\title{
Interactions between Curcumin Derivatives and Amyloid- $\beta$ Fibrils: Insights from Molecular Dynamics Simulations
}

\section{Supporting Information}

\author{
Joseph M. Jakubowski ${ }^{\dagger}$, Asuka A. Orr ${ }^{\dagger}$, Doan A. Le, Phanourios Tamamis* \\ Artie McFerrin Department of Chemical Engineering, Texas A\&M University, College Station, \\ Texas 77843-3122, United States \\ $\dagger$ Joseph M. Jakubowski and Asuka A. Orr are equally contributing first authors \\ *Correspondence: Phone: +1-979-862-1610; E-mail: tamamis@tamu.edu
}

The Supporting Information include additional details of the methods used in this study as well as supporting figures and tables. The Supporting Methods sections provides information regarding the simulations performed for the study as well as the methods used to analyze the simulation snapshots including pairwise molecule functional group : $A \beta_{1-42}$ residue interaction energy and molecule : $\mathrm{A} \beta_{1-42}$ fibril association energy calculations.

\section{Supporting Methods}

Supporting information on the methodology used in MD simulations: Each of the modeled systems were explicitly solvated in a water box that was constructed such that the edges of the water box were at least $15 \AA$ away from the fibril on all sides. As a cutoff of $12 \AA$ was used for the nonbonded interactions, we ensured that the $30 \AA$ distance between periodic images of the fibril was sufficient that no artificial "self-interaction" was present within the simulations, even when partial dissociation was observed. Water molecules were represented using the TIP3P water model. ${ }^{1}$ Histidines were neutral, in line with their protonation state at physiological $\mathrm{pH} .{ }^{2}$ Potassium and chloride ions were introduced to each simulation system at a concentration of 0.15 $\mathrm{M}$ with additional potassium or chloride ions introduced to neutralize the total charge of the simulation systems through 2000 steps of Monte Carlo simulations. ${ }^{3}$ The 0.15 M potassiumchloride ion concentration was used to model intracellular physiological conditions. ${ }^{4}$ Although the classical view is that $A \beta$ is deposited extracellularly, evidence from transgenic mice and human patients indicates that it can also accumulate intraneuronally, which may contribute to disease progression. $^{5}$

Each modeled system subsequently underwent 100 steps of steepest descent, 100 steps of Adopted Basis Newton-Raphson, and another 100 steps of steepest descent energy minimization with the $\mathrm{A} \beta_{1-42}$ fibril and molecules fixed and the ions constrained under $0.1 \mathrm{kcal} /\left(\mathrm{mol}-\AA^{2}\right)$ harmonic constraints. The systems were then subjected to 100 steps of steepest descent, 100 steps of Adopted Basis Newton-Raphson, and another 100 steps of steepest descent energy minimization with the $\mathrm{A} \beta \beta_{1-42}$ fibril backbone atoms and molecule heavy atoms constrained under $1.0 \mathrm{kcal} /\left(\mathrm{mol}-\AA^{2}\right)$ 
harmonic constraints and sidechain atoms constrained under $0.1 \mathrm{kcal} /\left(\mathrm{mol}-\AA^{2}\right)$ harmonic constraints. Each system was equilibrated under the same constraints for $1.0 \mathrm{~ns}$. Following equilibration, the constraints on the backbone and side chains were released and each system was simulated for an additional $100 \mathrm{~ns}$. Periodic boundary conditions were applied for all simulations. The temperature and pressure of all simulations were set to $300 \mathrm{~K}$ and $1 \mathrm{~atm}$, respectively.

Determination of the simulation stage per trajectory encompassing a molecule's binding mode leading to partial dissociation of the outermost peptide: Within the simulations in which partial $A \beta_{1-42}$ fibril dissociation of the outermost peptide is initiated (Table 1, column 3), the molecule does not necessarily remain in its initial docked pose prior or a posterior to partial dissociation. Rather the configuration and orientation of a molecule can fluctuate prior to the deformation of $\beta$-sheet interactions between the outermost peptide and its adjacent peptide within $\mathrm{A} \beta_{1-42}$ residue domain $12-20$, adopting specific binding modes (one per simulation) to initiate partial dissociation. Thus, to identify the specific binding modes, emphasis was given on determining the simulation stage in which the molecule remains relatively stable in complex with the $\mathrm{A} \beta_{1-42}$ fibril.

The simulation stage per trajectory was determined using an RMSD-based criterion coupled with visual inspection. The end of the simulation stage, or the point of partial dissociation, is defined as the last simulation snapshot in which all extended $\beta$-sheet interactions between the outermost peptide and its adjacent peptide within $A \beta_{1-42}$ residue domain 12-20 are permanently deformed. The beginning of the simulation stage of each binding mode leading to partial dissociation per trajectory is defined as the simulation snapshot in which the molecule's RMSD with respect to the final simulation stage snapshot (the point of partial dissociation) becomes and remains equal or less than $4 \AA$.

Additional information on structural and energetic analysis of the molecules' binding modes leading to partial $\mathbf{A} \boldsymbol{\beta}_{1-42}$ fibril dissociation: Calculation of molecule functional group : A $\beta_{1-42}$ residue pairwise interaction energy. The pairwise interaction energies between a molecule's functional groups and $A \beta_{1-42}$ residues for the simulation stage per trajectory were calculated using Eq. S2, with each energy term calculated through CHARMM. ${ }^{6}$

$$
\Delta G_{R R^{\prime}}^{\mathrm{inte}}=\frac{1}{f} \sum_{m \in f}\left(\sum_{i \in R} \sum_{j \in R^{\prime}}\left(E_{i j}^{\mathrm{Elec}}+E_{i j}^{\mathrm{GB}}\right)+\sum_{i \in R} \sum_{j \in R^{\prime}} E_{i j}^{\mathrm{vdW}}+\gamma \sum_{i \in R, R^{\prime}} \Delta\left(\mathrm{SASA}_{i}\right)\right)
$$

The first, second and third components of the equation above represent the polar, van der Waals and nonpolar solvation interaction energies between $R$ and $R^{\prime}$ respectively. $R$ corresponds to a given residue in the $A \beta_{1-42}$ fibril and $R^{\prime}$ corresponds to a given functional group of the molecule 
(Figure S1). The interaction energies of $m$ to $f$ frames corresponding to the duration of the simulation stage were summed and averaged.

The polar component of the total interaction energy $\left(\Delta G_{R R^{\prime}}^{\text {inte }}=G_{R R^{\prime}}^{\text {inte }} \quad G_{R^{\prime}} \quad G_{R}\right)$ is comprised of electrostatic interaction $\left(E_{i j}^{\mathrm{Elec}}\right)$ and generalized-Born $\left(E_{i j}^{\mathrm{GB}}\right)$ energy contributions between $\mathrm{A} \beta_{1-42}$ residue $R$ and functional group $R^{\prime}$. The polar component represents the interaction between $\mathrm{A} \beta_{1-42}$ residue $R$ and functional group $R^{\prime}$ and the interaction between residue $\mathrm{A} \beta_{1-42} R$ and the solvent polarization potential induced by functional group $R^{\prime}$. The nonpolar component (sum of the second and third term) consists of the van der Waals interactions between $\mathrm{A} \beta_{1-42}$ residue $R$ and functional group $R^{\prime}$ and the change in the nonpolar solvation energy due to binding $\left(\gamma \cdot \Delta \mathrm{SASA}_{i}\right)$. The nonpolar interaction energy term represents the nonpolar interactions with the surrounding solvent and cavity contributions.

The solvation terms were determined using the GBSW generalized-Born model. ${ }^{7}$ These calculations were executed with the nonpolar surface tension coefficient, $\gamma$, set to the default value of $0.03 \mathrm{kcal} \mathrm{mol}^{-1} \AA^{-2}$. The generalized-Born energy contribution $\left(E_{i j}^{\mathrm{GB}}\right)$ and solvent accessible surface area $\left(\triangle \mathrm{SASA}_{i}\right)$ are affected by the location of $R$ and $R^{\prime}$ in the complex. ${ }^{8}$ To compute the $E_{i j}^{\mathrm{GB}}$ term in Eq. 1, all atoms were included, and the charges of atoms outside the groups $R R^{\prime}, R^{\prime}$,

and $R$ were set to zero in each calculation of the terms $G_{R R^{\prime}}^{\text {inte }}, G_{R^{\prime}}$, and $G_{R}$, respectively. The $\triangle \mathrm{SASA}_{i}$ term expresses the difference in solvent accessible surface areas of the modeled $\mathrm{A} \beta_{1-42}$ residue $R$ and functional group $R^{\prime}$ within the complex and in the unbound states. The nonpolar interaction energy term represents the creation of a cavity in the surrounding solvent to accommodate molecules. For these calculations, we used infinite cutoff values.

For all simulations of a molecule in complex with the $A \beta_{1-42}$ fibril, we calculated the average polar and nonpolar interaction energy for each molecule functional group- $\mathrm{A} \beta_{1-42}$ residue pair within the simulation stage. Representative structural and interaction energy maps can be seen in Figures $S 9$, S10, S11, S12, S13, S14. In these figures, representative "head" and "tail" interactions of each mode are outlined in red, dotted lines and blue solid lines, respectively. ${ }^{9}$

Additional information on the calculation of association energies between the simulated molecules in complex with the A $\beta_{1-42}$ fibril using the MM-GBSA approximation: The binding energy of the molecules in complex with the $A \beta_{1-42}$ fibril was determined using two approaches, one of which was the Molecular Mechanics Generalized Born Surface Area (MM-GBSA) approximation as described by Eq. S3 below:

$$
\Delta G=G_{F C}-G_{F}-G_{C}
$$


where $G_{\mathrm{FC}}, G_{\mathrm{F}}$, and $G_{\mathrm{C}}$ represent the energies of the molecule : $\mathrm{A} \beta_{1-42}$ fibril complex, the $\mathrm{A} \beta_{1-42}$ fibril alone, and the molecule alone, respectively. We performed these calculations under the onetrajectory approximation, which assumed that the individual components of the molecule-A $\beta_{1-42}$ fibril complex in their bound state adopt the same conformation in their free states. Each individual energy contribution was estimated using the MM-GBSA approximation through Eq. S4 shown below:

$$
G=E^{\mathrm{Bonded}}+E^{\mathrm{Elec}}+E^{\mathrm{GB}}+E^{\mathrm{vdW}}+\gamma(\mathrm{SASA})
$$

Eq. S4

where $E^{\mathrm{Bonded}}, E^{\mathrm{Elec}}, E^{\mathrm{GB}}, E^{\mathrm{vdW}}$, and SASA represent the bonded energy, electrostatic interaction energy, generalized-Born energy, van der Waals energy, and solvent-accessible surface area of the system, respectively. Due to the one-trajectory approximation, the bonded term is subtracted out in the association energy calculation (Eq. S3). The polar contributions to the total MM-GBSA association energy were expressed by the sum of the electrostatic interaction energy and the generalized-Born energy. The nonpolar contributions to the total MM-GBSA association energy comprised of the van der Waals energy and solvent-accessible surface area. We used the GBSW generalized-Born implicit solvent model with the nonpolar surface tension coefficient, $\gamma$, set to the default value $\left(0.03 \mathrm{kcal} \mathrm{mol}^{-1} \AA^{-2}\right)$. The cutoffs used for these calculations were infinite. All energy calculations were performed in CHARMM. ${ }^{6}$

\section{Supporting Results}

Conformational analysis of the modeled $A \beta_{1-42}$ fibril within the simulations: We calculated the RMSD of the $A \beta_{1-42}$ fibril's residue domains ${ }_{12} \mathrm{VHHQKLVFF}_{20},{ }_{16} \mathrm{KLVFFA}_{21},{ }_{2} \mathrm{GAIIG}_{33}$, and ${ }_{32} \mathrm{IGLMVGG}_{38}$ with respect to the initial structure in each case for the entire simulation trajectories, by averaging across simulations in which partial dissociation was observed (Figure S2A) or simulations in which no partial dissociation was observed (Figure S2B), or simulations of the uncomplexed $A \beta_{1-42}$ fibril (Figure S2C). RMSD deviations are relatively low for all domains in the uncomplexed $A \beta_{1-42}$ fibril simulations, whereas the largest RMSD deviations can be observed within the ${ }_{12} \mathrm{VHHQKLVFF}_{20}$ and ${ }_{16} \mathrm{KLVFFA}_{21}$ overlapping domains in the simulations in which partial dissociation is initiated. The RMSF values associated with the same domains are relatively larger in simulations in which partial dissociation is initiated (Figure S4-S6). The corresponding deviations of the two overlapping domains are slightly larger in simulations in which no partial dissociation was observed compared to the uncomplexed fibril simulations, which could be a possible outcome of the two domains slight re-adjustment due to interactions with the molecules, and/or the loss of a portion of $\beta$-bridges of the outermost peptide in specific simulations.

Determination of the stability of the bound molecules in complex with the modeled A $\boldsymbol{\beta}_{1-42}$ fibril within the simulations: Within a miniscule subset of simulations ( 2 out of 94 simulations), 
the docked molecule loses contacts with the $A \beta_{1-42}$ fibril and is no longer in the vicinity of the fibril for the remainder of the simulations. In simulations of SY12 or SY31 in complex with the $\mathrm{A} \beta_{1-42}$ fibril, there were no instances in which the molecules lose contact with the fibril. In simulations of SY5 and curcumin, independently, there was one instance in which the molecule loses contact with the fibril. However, in the vast majority of the simulations, the molecules remain bound to the fibril for the full $100 \mathrm{~ns}$ duration of the simulation. Additionally, within simulations in which the molecules remain bound to the fibril, the molecules may adopt partially unstable binding modes in the vicinity of the fibril. Importantly, in the vast majority of the simulations, the molecules adopt stable binding modes (Table 1, column 3).

Binding energy calculations: We used two independent scoring methods, the MM-GBSA approximation, ${ }^{10}$ and AutoDock Vina scoring function. ${ }^{11} \mathrm{We}$ first performed a linear regression between the values obtained by the two methods, through which we observed a high correlation per molecule, regardless of if the molecule in the simulations can lead or not to partial dissociation. The $R^{2}$ values obtained are 0.95 for SY12, 0.96 for SY31, 0.98 for SY5 and 0.99 for curcumin.

In addition, we extracted the simulation in which the bound molecule in complex with the $A \beta_{1-42}$ fibril acquired the lowest average binding energy for each molecule and each binding mode, using the two methods independently. Importantly, the simulations identified as acquiring the lowest binding energy per molecule and per binding mode were the same across the two methods, confirming consistency between the two.

Extended simulations of binding mode 3 leading to partial dissociation of the outermost peptide: In both extended simulations of SY12 and SY31 adopting binding mode $p d 3$, the $\beta$-sheet interactions between the first outermost peptide of the $A \beta_{1-42}$ fibril and the second outermost peptide are nearly completely lost.

SY31 adopts a conformation reminiscent of binding mode 2 upon disruption of the $\beta$-sheet interactions in the residue domain ${ }_{12} \mathrm{VHHQKLVFF}_{20}$ between the first and second outermost peptide. Despite that, the partial dissociation of the first outermost peptide progressed until nearly full dissociation: In the simulation of SY31, only $\beta$-sheet interactions between residues 39-41 of the first and second outermost peptides remain at the end of the $200 \mathrm{~ns}$ simulation.

SY12 remains in binding mode 3 for the full duration of the extended $200 \mathrm{~ns}$ MD simulation. The ability of SY12 to remain in binding mode 3 after the N-terminal of the first outermost peptide is dissociated may be attributed to the ability of its anchoring central $\mathrm{R}^{1}$ group to form hydrogen bonds to the backbone $\mathrm{N}$ of Gly ${ }^{33}$ within the $\mathrm{A} \beta_{1-42}$ fibril through its oxygen atom (Figure S20). Due to the enhanced stability of its central $\mathrm{R}^{1}$ group, SY12 remains in binding mode 3 for a sufficient time to fully disrupt the $\beta$-sheet interactions of the $\mathrm{C}$-terminal, residues $33-42$, such that eventually all $\beta$-sheet interactions in the first outermost peptide except for $\beta$-sheet interactions 
formed by $\mathrm{Asn}^{27}, \mathrm{Lys}^{28}, \mathrm{Gly}^{29}, \mathrm{Ala}^{30}, \mathrm{Ile}^{31}$, and $\mathrm{Ile}^{32}$ are deformed at the end of the $200 \mathrm{~ns}$ simulation. 


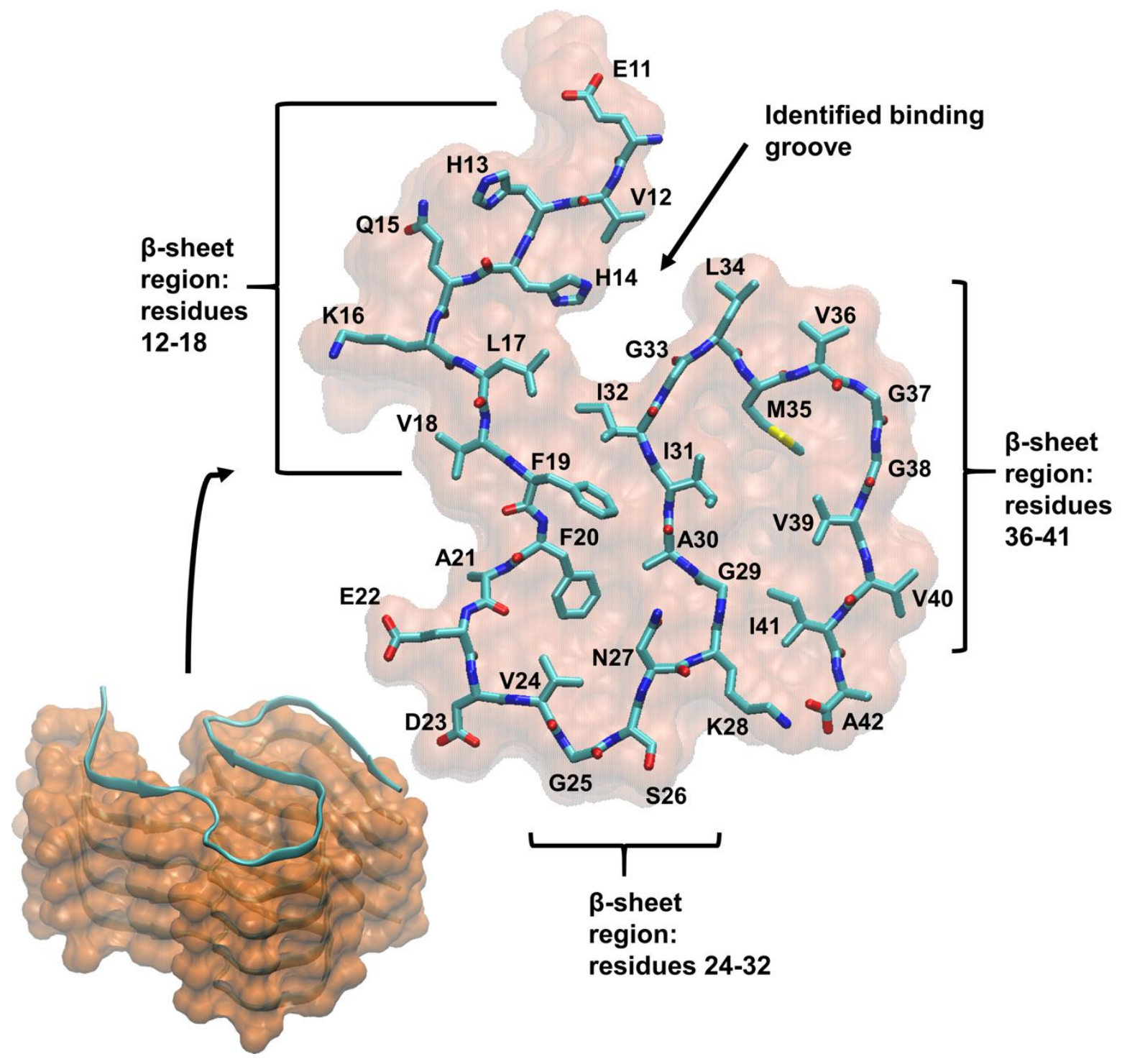

Figure S1: The structure of the modeled $A \beta_{1-42}$ fibril, with the 11-42 domain resolved, as characterized through ssNMR. ${ }^{9}$ The figure presents a side view of the fibril with sidechains to show the sidechain packing (top) and the corresponding surface representation of the fibril (bottom). All $\beta$-sheets regions are indicated in brackets (top). The identified binding groove of curcumin and its derivatives is indicated through a black arrow (top). 

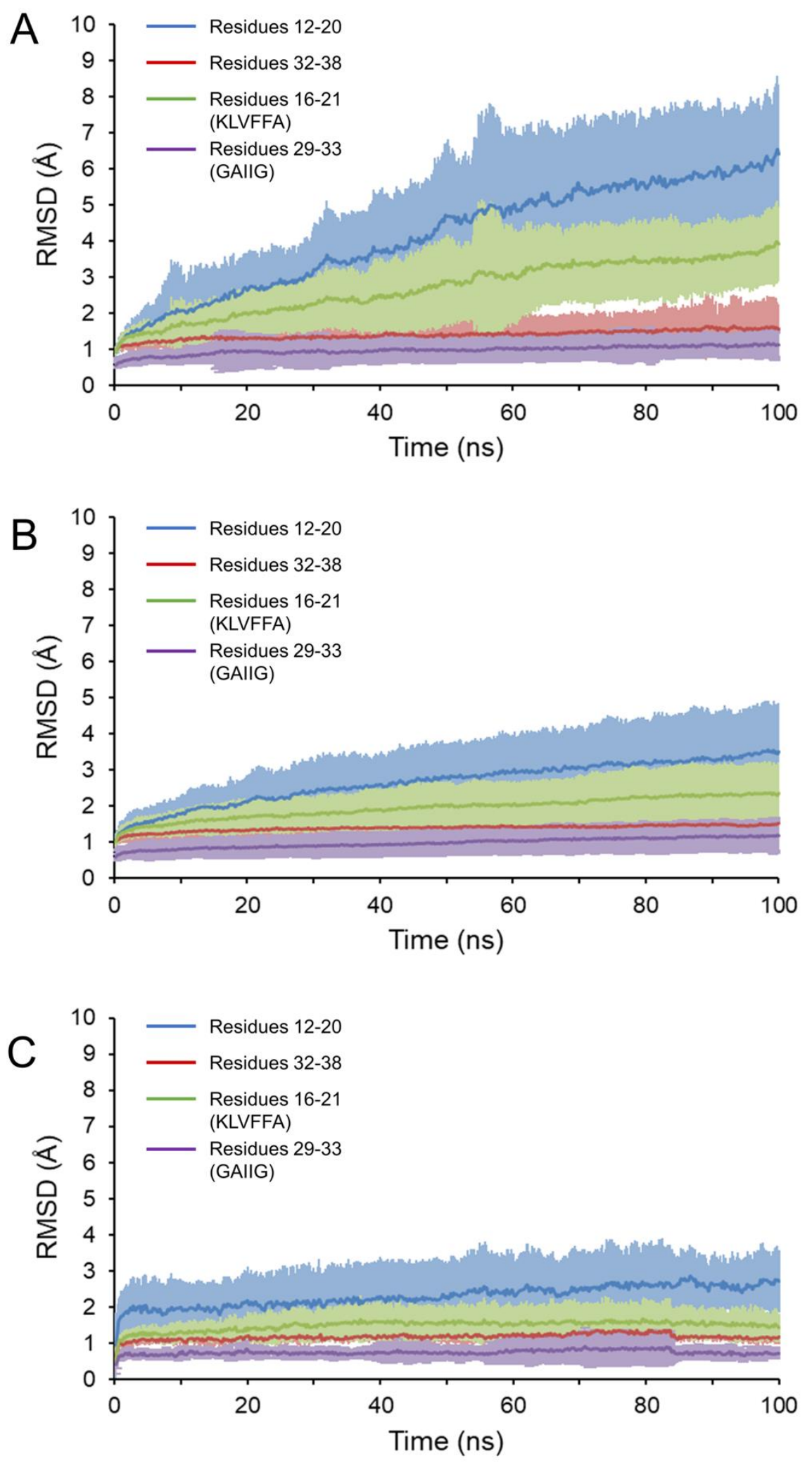
Figure S2: The average $A \beta_{1-42}$ fibril backbone RMSD of four key regions as a function of simulation time for (A) all simulations across all molecules in which partial dissociation of the outermost peptide of the $A \beta_{1-42}$ fibril is observed, (B) all simulations across all molecules in the absence of dissociation, and (C) all simulations of the $A \beta_{1-42}$ fibril in the absence of any molecule. The RMSD for each simulation was calculated with respect to each simulation's initial structure and was only calculated for the first $100 \mathrm{~ns}$ for fair comparison. The average backbone RMSD of residues 12-20 for the entire simulation is in blue. The average backbone RMSD of residues 32-38 for the entire simulation is in red. The average backbone RMSD of residues 16-21 for the entire simulation is in green. The average backbone RMSD of residues 29-33 for the entire simulation is in purple. 

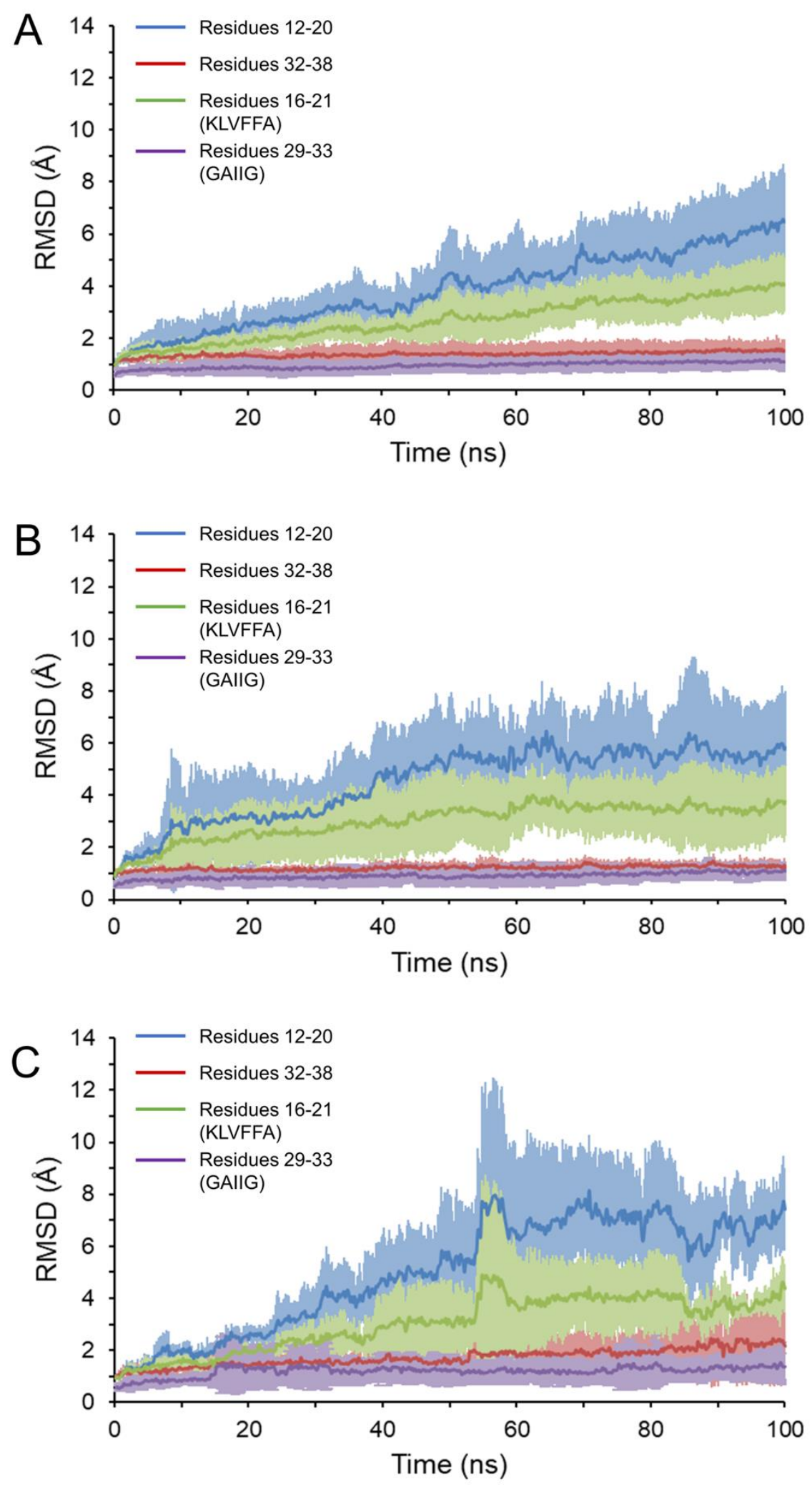
Figure S3: The average backbone RMSD of four key regions as a function of simulation time from (A) all simulations across all molecules in which the molecule adopts binding mode $p d 1$, (B) all simulations across all molecules in which the molecule adopts binding mode $p d 2$, and (C) all simulations across all molecules in which the molecule adopts binding mode $p d 3$. The RMSD for each simulation was calculated with respect to each simulation's initial structure and was only calculated for the first $100 \mathrm{~ns}$ for fair comparison. The average backbone RMSD of residues 12-20 for the entire simulation is in blue. The average backbone RMSD of residues $32-38$ for the entire simulation is in red. The average backbone RMSD of residues 16-21 for the entire simulation is in green. The average backbone RMSD of residues $29-33$ for the entire simulation is in purple. 

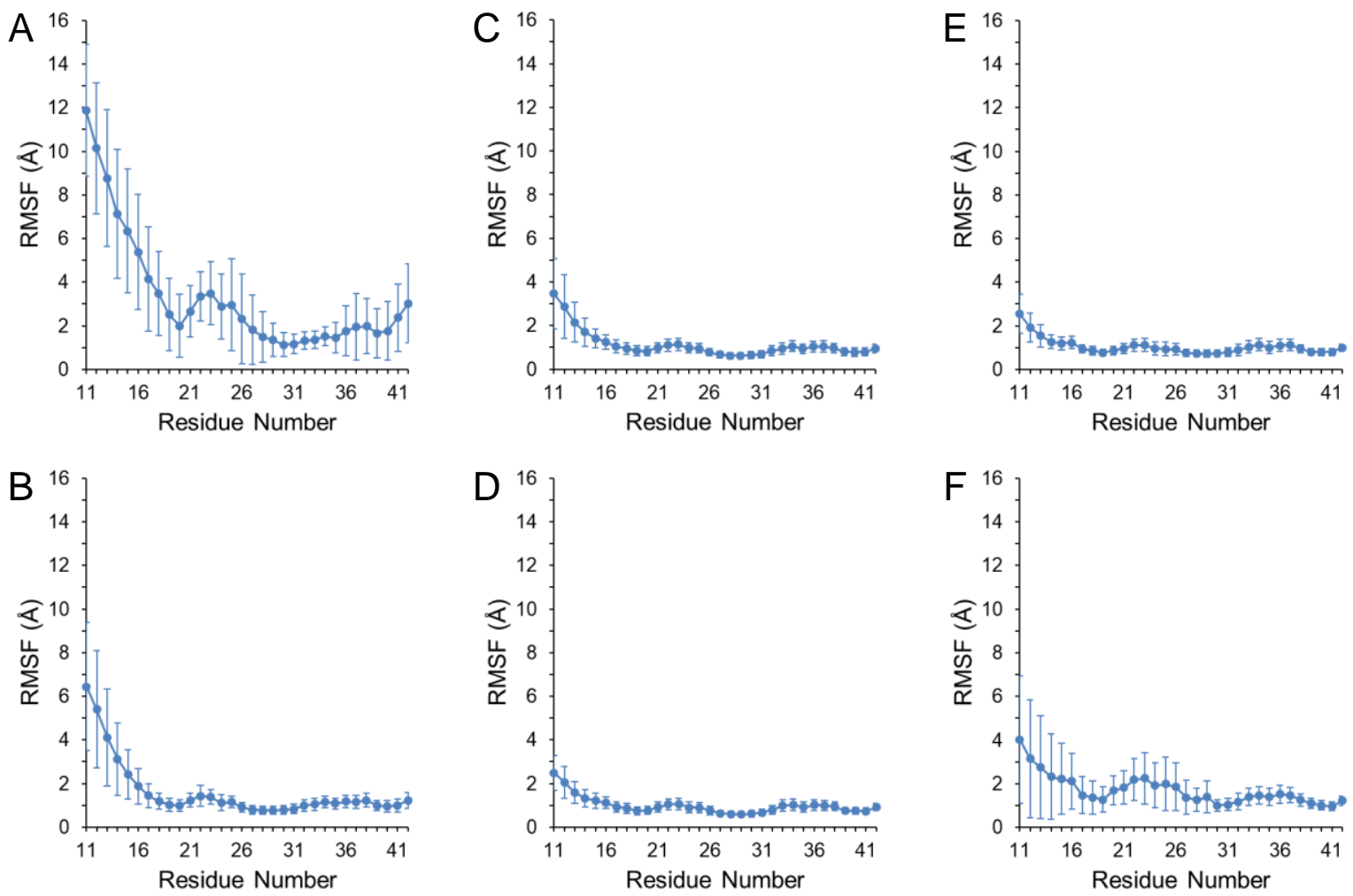

Figure S4: The average RMSF of $\mathrm{C} \alpha$ atoms per residues of the (A) first outermost peptide, (B) second outermost peptide, (C) third outermost peptide, (D) fourth outermost peptide, (E) fifth outermost peptide, and (F) sixth outermost peptide of the $A \beta_{1-42}$ fibril in all simulations across all molecules in which partial dissociation of the outermost peptide is observed. The results correspond to the first $100 \mathrm{~ns}$ of each simulation, irrespective if specific runs were extended, for fair analysis and comparison purposes. 

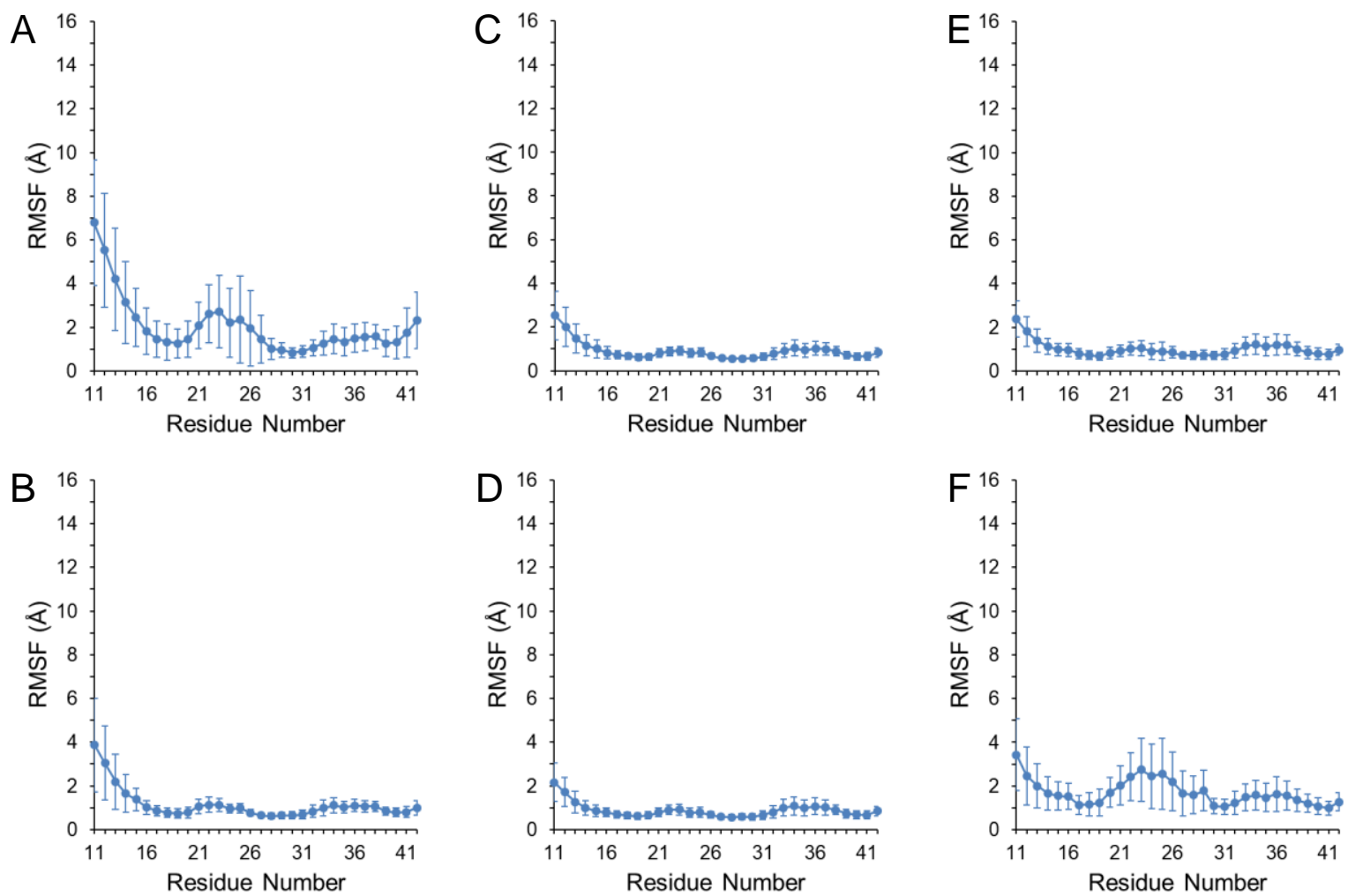

Figure S5: The average RMSF of $\mathrm{C} \alpha$ atoms per residues of the (A) first outermost peptide, (B) second outermost peptide, (C) third outermost peptide, (D) fourth outermost peptide, (E) fifth outermost peptide, and $(F)$ sixth outermost peptide of the $A \beta_{1-42}$ fibril in all simulations across all molecules in which no partial dissociation is observed. The results correspond to the first $100 \mathrm{~ns}$ of each simulation, irrespective if specific runs were extended, for fair analysis and comparison purposes. 

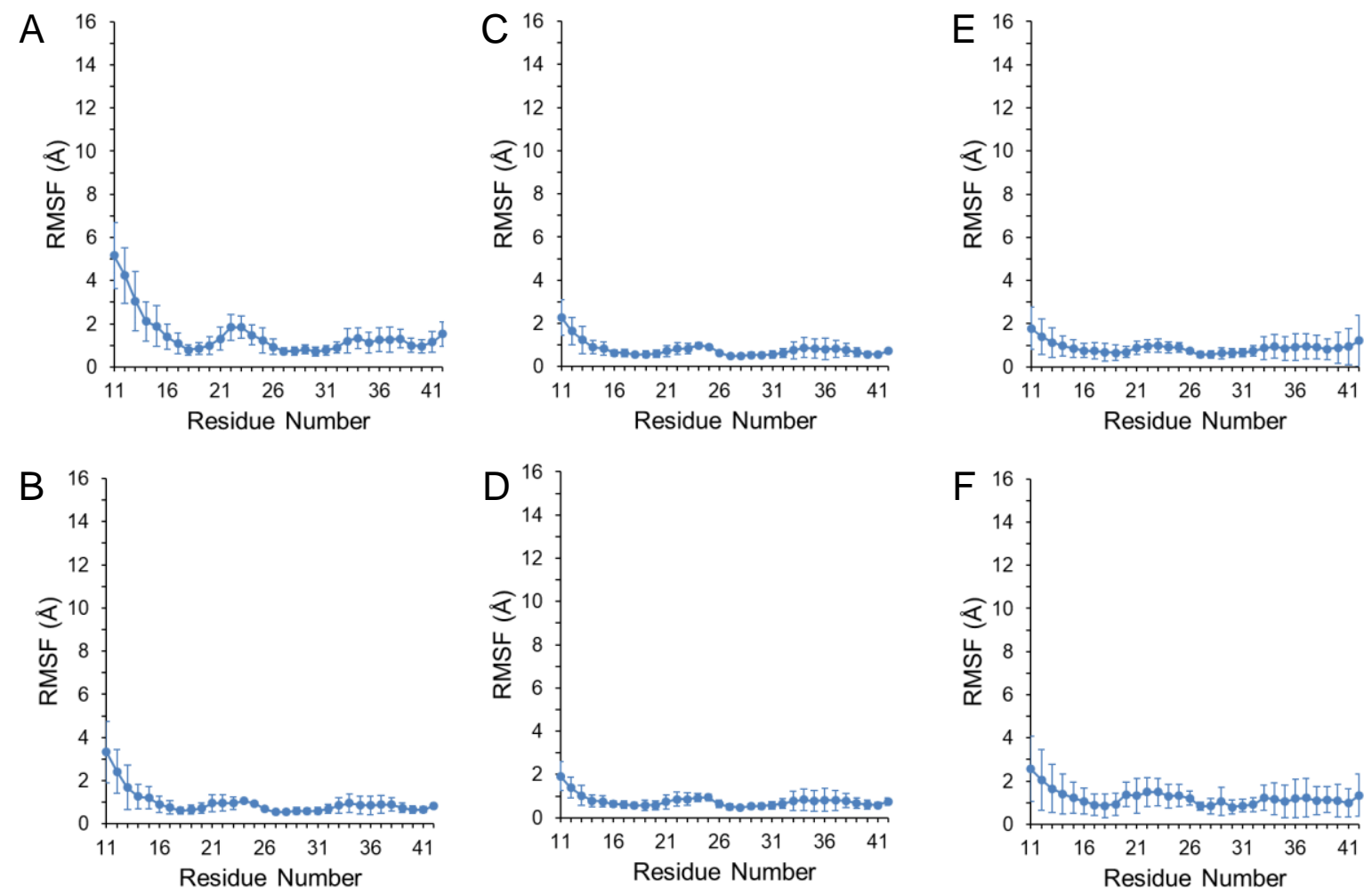

Figure S6: The average RMSF of $\mathrm{C} \alpha$ atoms per residues of the (A) first outermost peptide, (B) second outermost peptide, (C) third outermost peptide, (D) fourth outermost peptide, (E) fifth outermost peptide, and (F) sixth outermost peptide of the $A \beta_{1-42}$ fibril in all simulations in the absence of any molecule. The results correspond to the first $100 \mathrm{~ns}$ of each simulation, irrespective if specific runs were extended, for fair analysis and comparison purposes. 

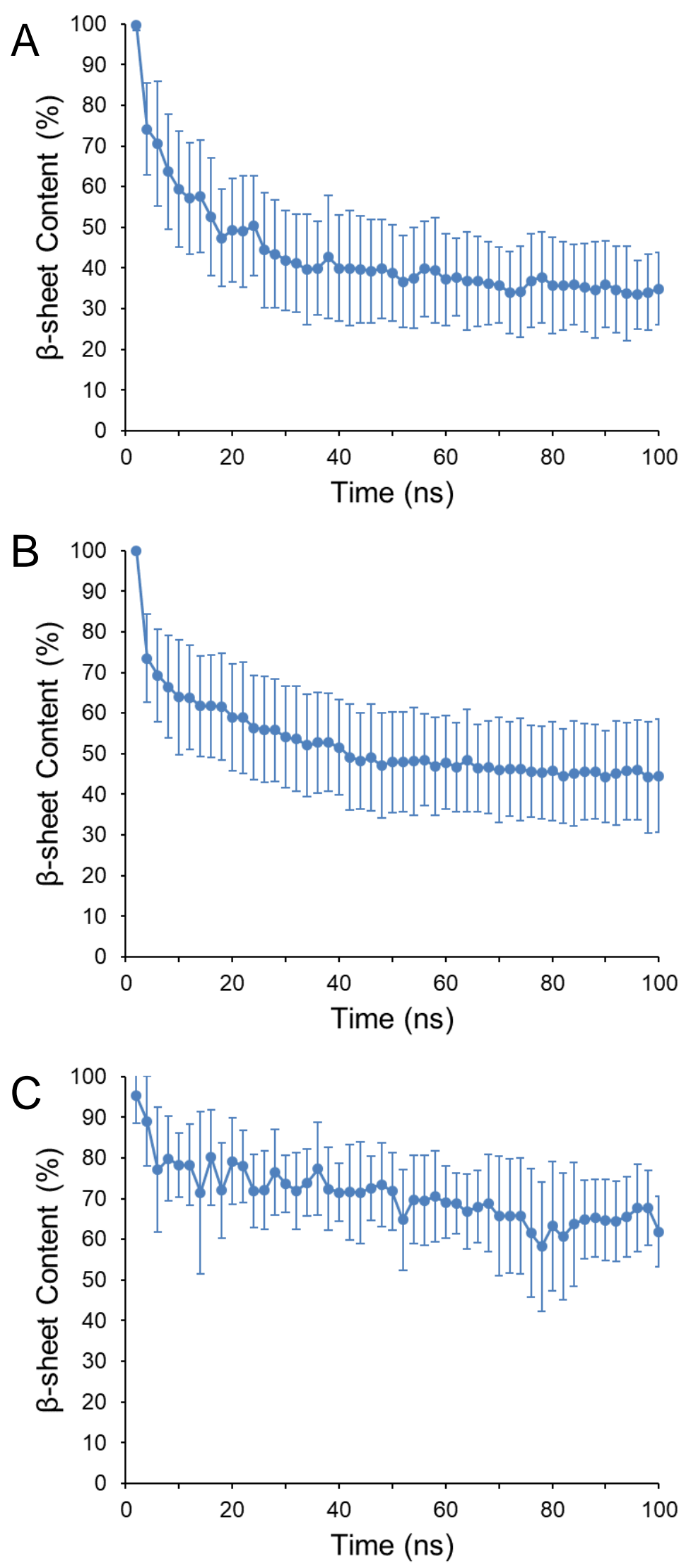
Figure S7: The average percentage of $\beta$-sheet content as a function of simulation time associated with the first outermost peptide of the $A \beta_{1-42}$ fibril in (A) all simulations across all molecules in which partial dissociation of the $A \beta_{1-42}$ fibril is observed, (B) all simulations across all molecules in which no partial dissociation of the $A \beta_{1-42}$ fibril is observed, and (C) all simulations of the $A \beta_{1-42}$ fibril in the absence of any molecule. We calculated the $\beta$-sheet content of each simulation corresponding to the first $100 \mathrm{~ns}$ for fair analysis and comparison purposes. 


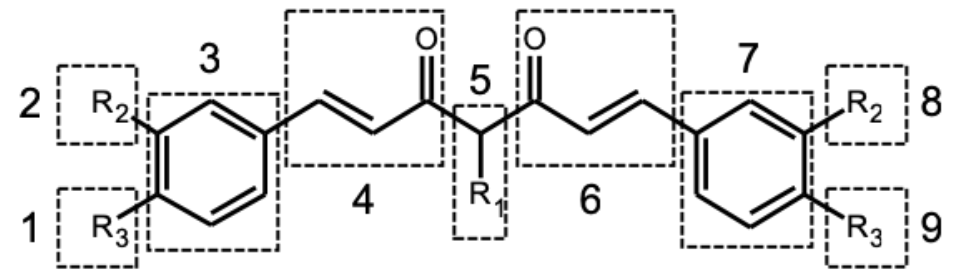

Figure S8: Decomposition of the molecules into functional groups used for the structural and energetic analysis. 

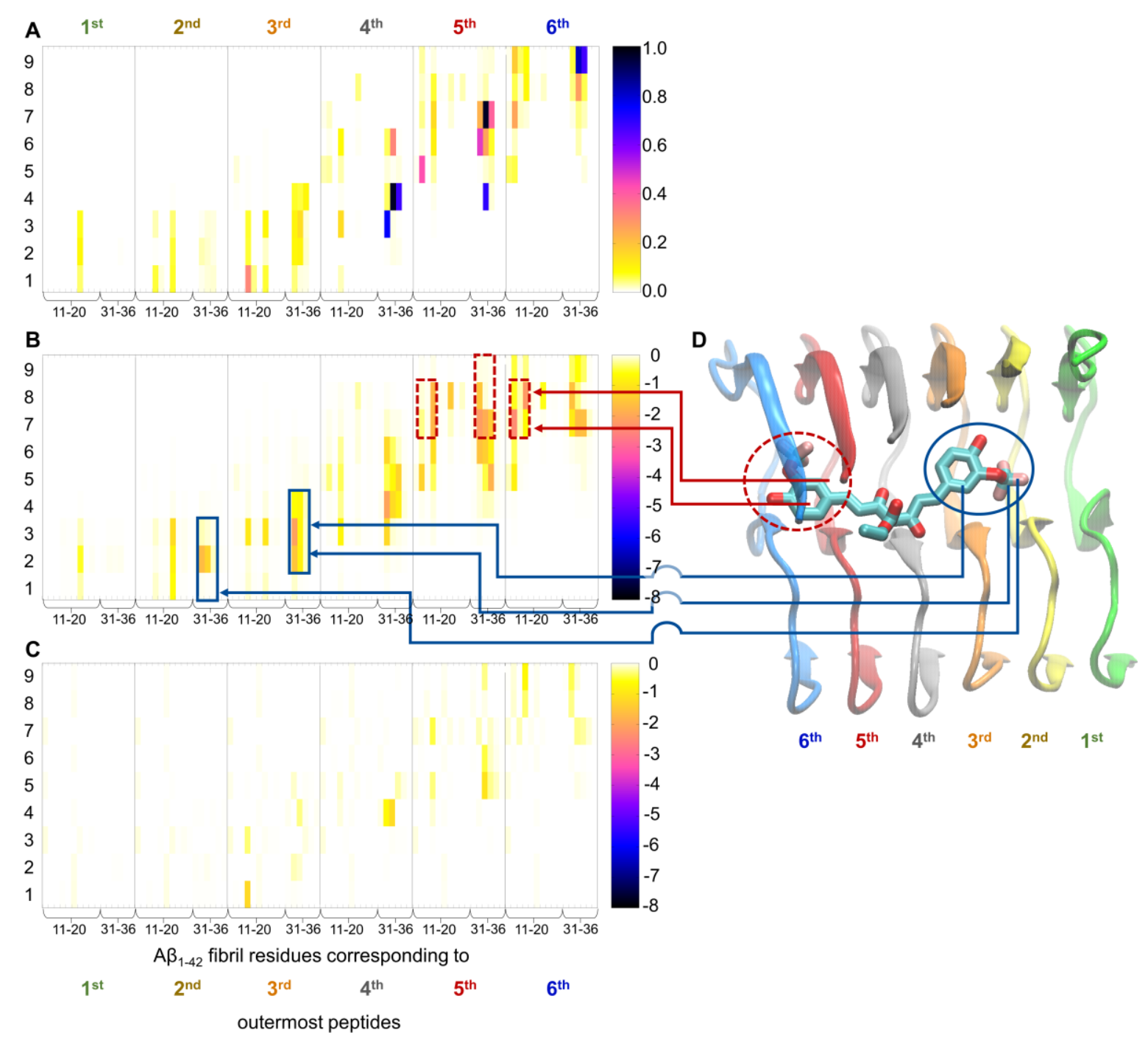

Figure S9: (A) Contact and (B, C) interaction energy maps calculated for the entire simulation representative of binding mode $n d 1$, corresponding to SY12 in complex with an A $\beta_{1-42}$ fibril that adopts the lowest energy binding mode $n d 1$. The corresponding molecular graphics image is presented in panel (D) and Figure 4A of the main text. The $\mathrm{x}$-axis corresponds to the $\mathrm{A} \beta_{1-42}$ fibril residues for the first, second, third, fourth, fifth, and sixth outermost peptides (shown in top and bottom only). The y-axis corresponds to the different functional groups of the molecules as described in Figure S2. The colored palette on the right of each panel corresponds to (A) the probability of contact between a molecule's functional group and an $A \beta_{1-42}$ residue side chain, and the average interaction energy of (B) nonpolar and (C) polar interactions between a molecule's functional group and an $A \beta_{1-42}$ residue. Interactions indicating the head (one aromatic functional group and its substituents) of binding mode $n d 1$ are outlined by red, dotted lines. Interactions indicating the tail (remaining aromatic functional group and its substituents) of binding mode $n d 1$ are outlined by solid blue lines. Despite that this map corresponds to SY12 in complex with an $A \beta_{1-42}$ fibril that adopts the lowest energy binding mode $n d 1$, it is considered to be representative of other calculated maps of all molecules adopting binding mode $n d 1$ (i.e., the indicated interactions are common across all maps). 


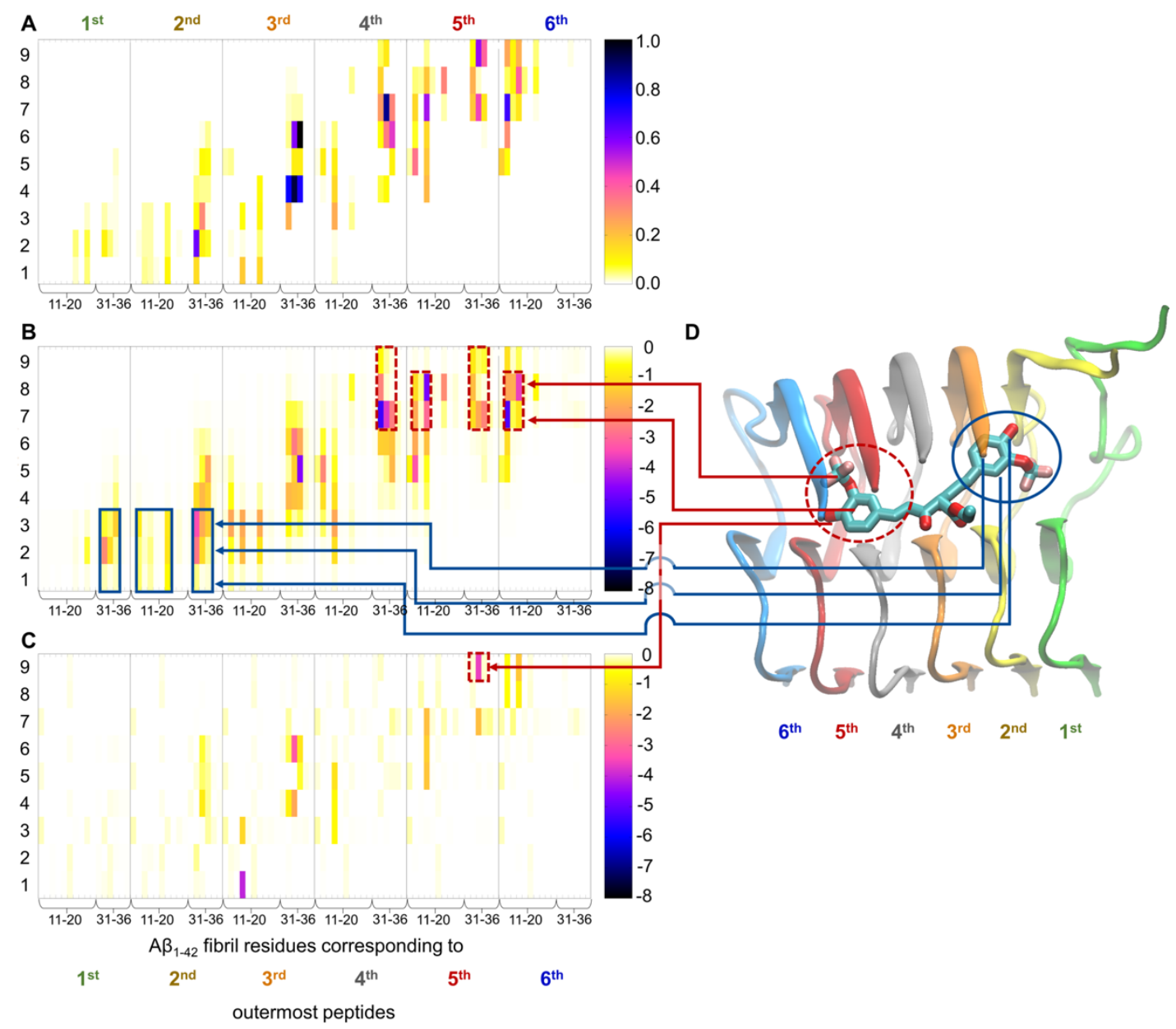

Figure S10: (A) Contact and (B, C) interaction energy maps calculated for a simulation stage leading to partial dissociation representative of binding mode $p d 1$, corresponding to SY12 in complex with an $A \beta_{1-42}$ fibril that adopts the lowest energy binding mode $p d 1$. The corresponding molecular graphics image is presented in panel (D) and Figure 4D of the main text. The $\mathrm{x}$-axis corresponds to the $\mathrm{A} \beta_{1-42}$ fibril residues for the first, second, third, fourth, fifth, and sixth outermost peptides (shown in top and bottom only) given that partial dissociation is initiated by the deformation of interactions between the first outermost peptide and its adjacent (second) peptide in the fibril. The y-axis corresponds to the different functional groups of the molecules as described in Figure S2. The colored palette on the right of each panel corresponds to (A) the probability of contact between a molecule's functional group and an $A \beta_{1-42}$ residue side chain, and the average interaction free energy of $(B)$ nonpolar and $(C)$ polar interactions between a molecule's functional group and an $A \beta_{1-42}$ residue. Interactions indicating the anchor (one aromatic functional group and its substituents) of binding mode $p d 1$ are outlined by red, dotted lines. Interactions indicating the breaker (remaining aromatic functional group and its substituents) of binding mode $p d 1$ are outlined by solid blue lines. Despite that this map corresponds to a specific simulated stage and that this map corresponds to SY12 in complex with an $\mathrm{A} \beta_{1-42}$ fibril that adopts the lowest energy binding mode $p d 1$, it is considered to be representative of other calculated maps of all molecules leading to partial dissociation through binding mode $p d 1$ (i.e., the indicated interactions are common across all maps). 


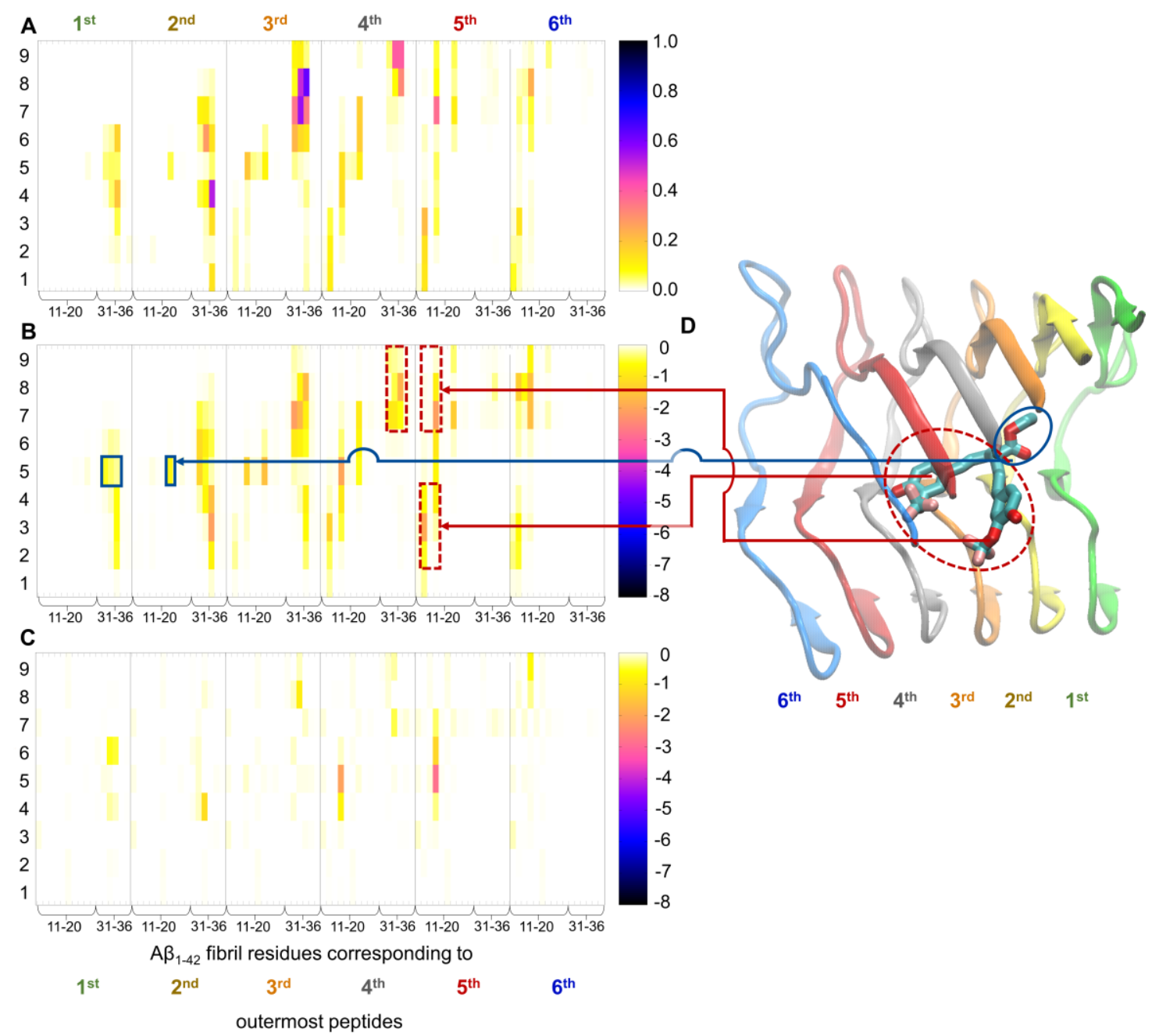

Figure S11: (A) Contact and (B, C) interaction energy maps calculated for the entire simulation representative of binding mode $n d 2$, corresponding to SY12 in complex with an A $\beta_{1-42}$ fibril that adopts the lowest energy binding mode $n d 2$. The corresponding molecular graphics image is presented in panel (D) and Figure 5A of the main text. The $x$-axis corresponds to the $A \beta_{1-42}$ fibril residues for the first, second, third, fourth, fifth, and sixth outermost peptides (shown in top and bottom only). The y-axis corresponds to the different functional groups of the molecules as described in Figure S2. The colored palette on the right of each panel corresponds to (A) the probability of contact between a molecule's functional group and an $A \beta_{1-42}$ residue side chain, and the average interaction energy of (B) nonpolar and (C) polar interactions between a molecule's functional group and an $A \beta_{1-42}$ residue. Interactions indicating the head (both aromatic functional groups and their substituents) of binding mode $n d 2$ are outlined by red, dotted lines. Interactions indicating the tail (central $\mathrm{R}^{1}$ group) of binding mode $n d 2$ are outlined by solid blue lines. Despite that this map corresponds to SY12 in complex with an $\mathrm{A} \beta_{1-42}$ fibril that adopts the lowest energy binding mode $n d 2$, it is considered to be representative of other calculated maps of all molecules adopting binding mode $n d 2$ (i.e., the indicated interactions are common across all maps). 


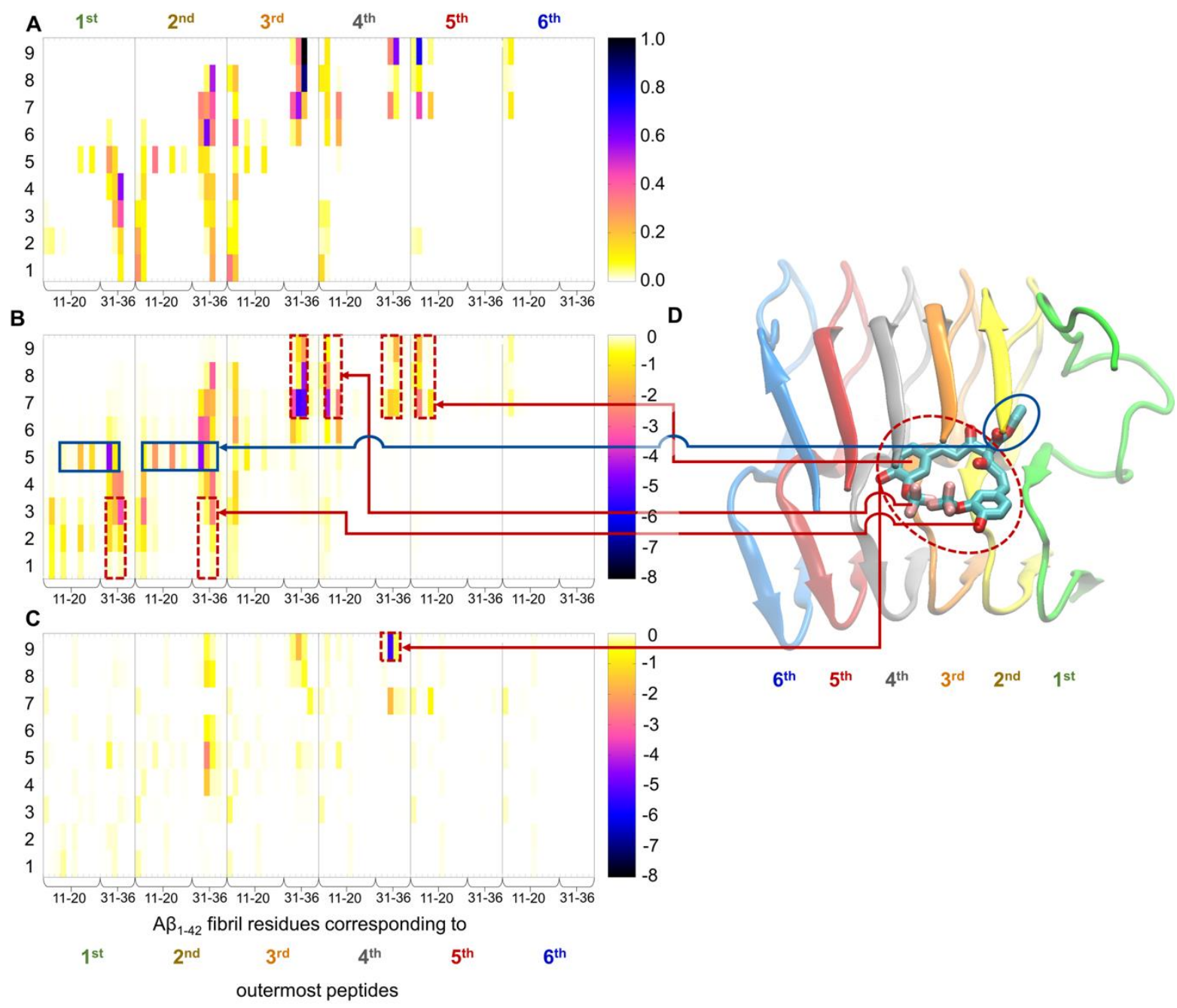

Figure S12: (A) Contact and (B, C) interaction energy maps calculated for a simulation stage leading to partial dissociation representative of binding mode $p d 2$, corresponding to SY12 in complex with an $\mathrm{A} \beta_{1-42}$ fibril that adopts the lowest energy binding mode $p d 2$. The corresponding molecular graphics image is presented in panel (D) and Figure 5D of the main text. The $\mathrm{x}$-axis corresponds to the $\mathrm{A} \beta_{1-42}$ fibril residues for the first, second, third, fourth, fifth, and sixth outermost peptides (shown in top and bottom only) given that partial dissociation is initiated by the deformation of interactions between the first outermost peptide and its adjacent (second) peptide in the fibril. The y-axis corresponds to the different functional groups of the molecules as described in Figure S2. The colored palette on the right of each panel corresponds to (A) the probability of contact between a molecule's functional group and an $A \beta_{1-42}$ residue side chain, and the average interaction energy of (B) nonpolar and (C) polar interactions between a molecule's functional group and a $A \beta_{1-42}$ residue. Interactions indicating the anchor (both aromatic functional groups and their substituents) of binding mode $p d 2$ are outlined by red, dotted lines. Interactions indicating the breaker (central $\mathrm{R}^{1}$ group) of binding mode $p d 2$ are outlined by solid blue lines. Despite that this map corresponds to a specific simulated stage and that this map corresponds to SY12 in complex with an $A \beta_{1-42}$ fibril that adopts the lowest energy binding mode $p d 2$, it is considered to be representative of other calculated maps of all molecules leading to partial dissociation through binding mode $p d 2$ (i.e., the indicated interactions are common across all maps). 


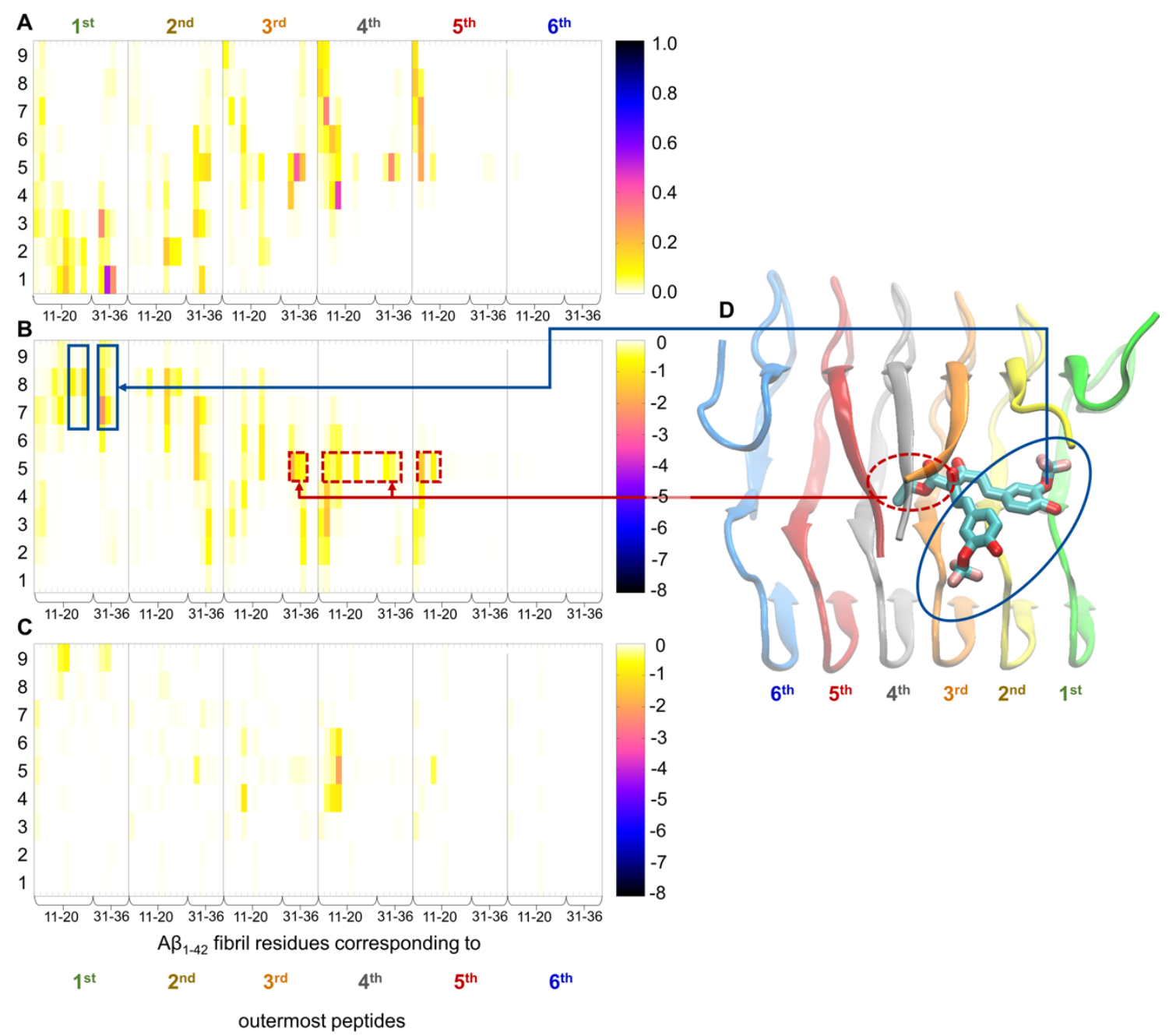

Figure S13: (A) Contact and (B, C) interaction energy maps calculated for the entire simulation representative of binding mode $n d 3$, corresponding to SY12 in complex with an $\mathrm{A} \beta_{1-42}$ fibril that adopts the lowest energy binding mode $n d 3$. The corresponding molecular graphics image is presented in panel (D) and Figure 6A of the main text. The $x$-axis corresponds to the $A \beta_{1-42}$ fibril residues for the first, second, third, fourth, fifth, and sixth outermost peptides (shown in top and bottom only). The y-axis corresponds to the different functional groups of the molecules as described in Figure $\mathrm{S} 2$. The colored palette on the right of each panel corresponds to (A) the probability of contact between a molecule's functional group and an $A \beta_{1-42}$ residue side chain, and the average interaction energy of (B) nonpolar and (C) polar interactions between a molecule's functional group and an $A \beta_{1-42}$ residue. Interactions indicating the head (central $\mathrm{R}^{1}$ group) of binding mode $n d 3$ are outlined by red, dotted lines. Interactions indicating the tail (both aromatic functional groups and their substituents) of binding mode $n d 3$ are outlined by solid blue lines. Despite that this map corresponds to SY12 in complex with an $\mathrm{A} \beta_{1-42}$ fibril that adopts the lowest energy binding mode $n d 3$, it is considered to be representative of other calculated maps of all molecules adopting binding mode $n d 3$ (i.e., the indicated interactions are common across all maps). 


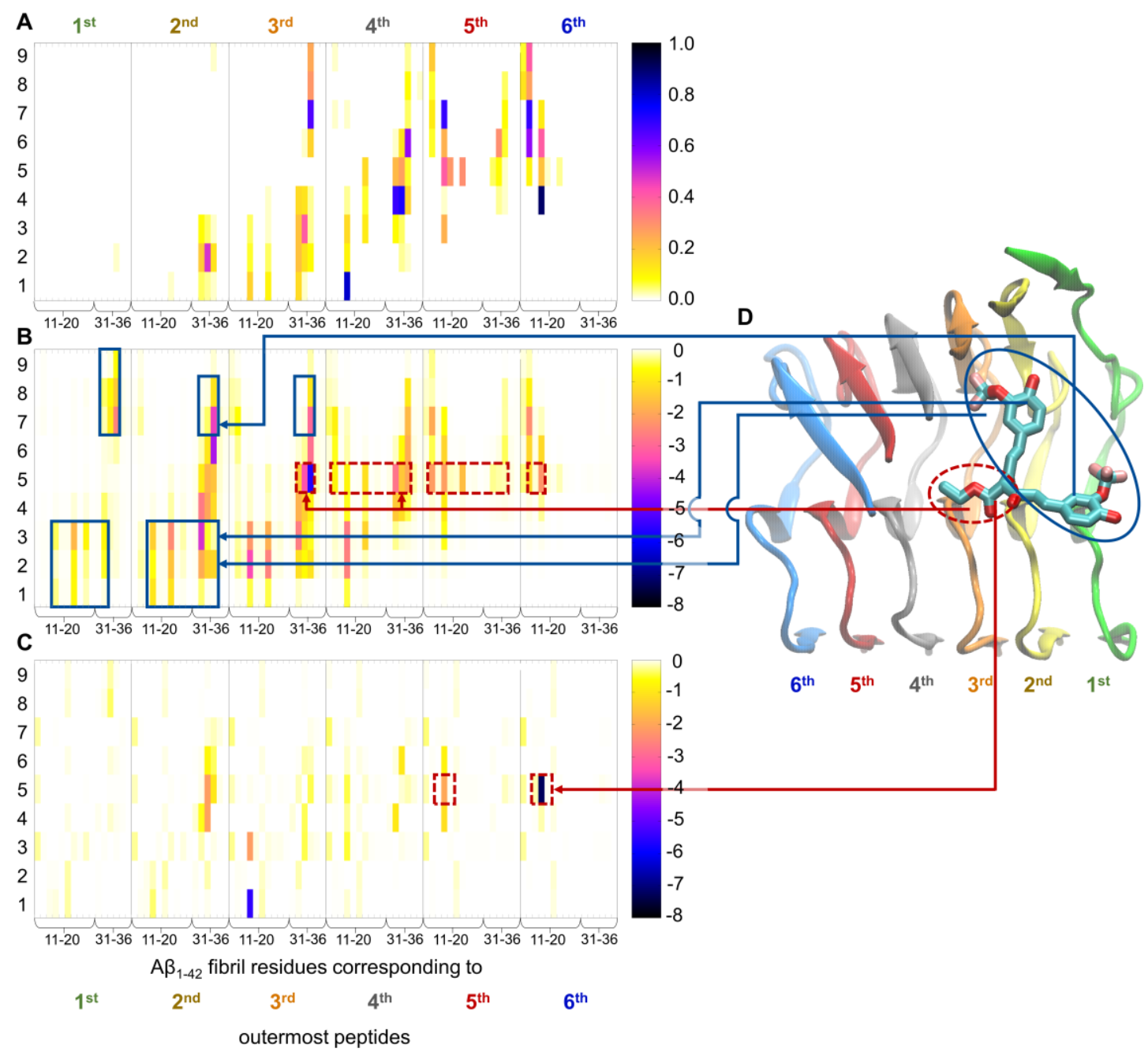

Figure S14: (A) Contact and (B, C) interaction energy maps calculated for a simulation stage leading to partial dissociation representative of binding mode $p d 3$, corresponding to SY12 in complex with an $\mathrm{A} \beta_{1-42}$ fibril that adopts the lowest energy binding mode $p d 3$. The corresponding molecular graphics image is presented in panel (D) and Figure 6D of the main text. The $\mathrm{x}$-axis corresponds to the $\mathrm{A} \beta_{1-42}$ fibril residues (shown in bottom only) for the first, second, third, fourth, fifth, and sixth outermost peptides (shown in bottom only) given that partial dissociation is initiated by the deformation of interactions between the first outermost peptide and its adjacent (second) peptide in the fibril. The y-axis corresponds to the different functional groups of the molecules as described in Figure S2. The colored palette on the right of each panel corresponds to (A) the probability of contact between a molecule's functional group and an $A \beta_{1-42}$ residue side chain, and the average interaction energy of (B) nonpolar and (C) polar interactions between a molecule's functional group and an $A \beta_{1-42}$ residue. Interactions indicating the anchor (central $\mathrm{R}^{1}$ group) of binding mode $p d 3$ are outlined by red, dotted lines. Interactions indicating the breaker (both aromatic functional groups and their substituents) of binding mode $p d 3$ are outlined by solid blue lines. Despite that this map corresponds to a specific simulated stage and that this map corresponds to SY12 in complex with an $\mathrm{A} \beta_{1-42}$ fibril that adopts the lowest energy binding mode $p d 3$, it is considered to be representative of other calculated maps of all molecules leading to partial dissociation through binding mode $p d 3$ (i.e., the indicated interactions are common across all maps). 

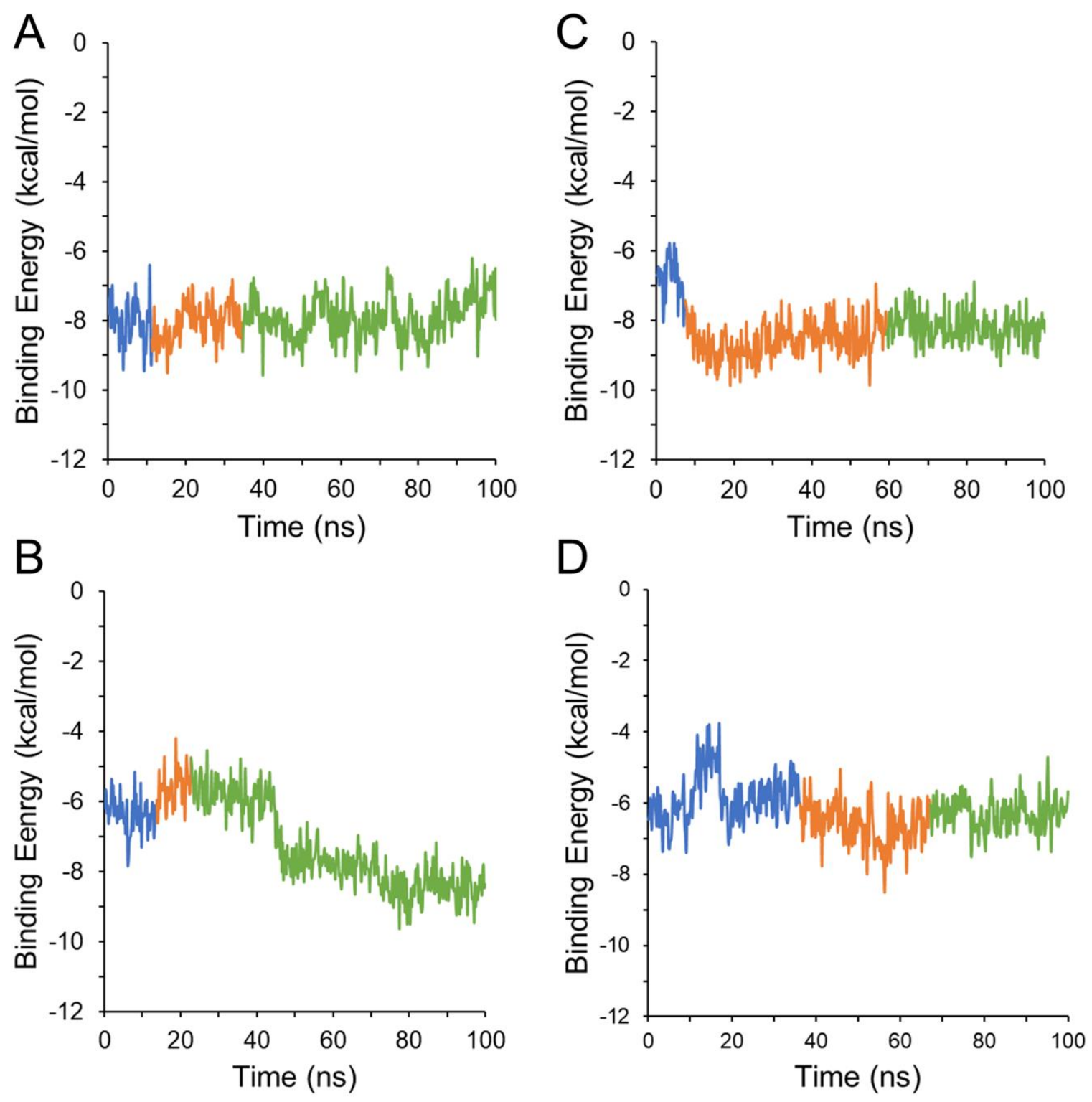

Figure S15: The binding energy (calculated through AutoDock Vina scoring function) between the molecule and the $\mathrm{A} \beta_{1-42}$ fibril as a function of simulation time for (A) SY12, (B) SY31, (C) SY5, and (D) curcumin. The plotted binding energy was calculated from simulations in which the molecules acquire the lowest average binding energy, per molecule, and adopt binding mode $p d 1$. Blue represents the binding energy prior to the simulation stage corresponding to the respective simulation leading to partial dissociation. Orange represents the binding energy during the simulation stage leading to partial dissociation. Green represents the binding energy posterior to the simulation stage leading to partial dissociation. 

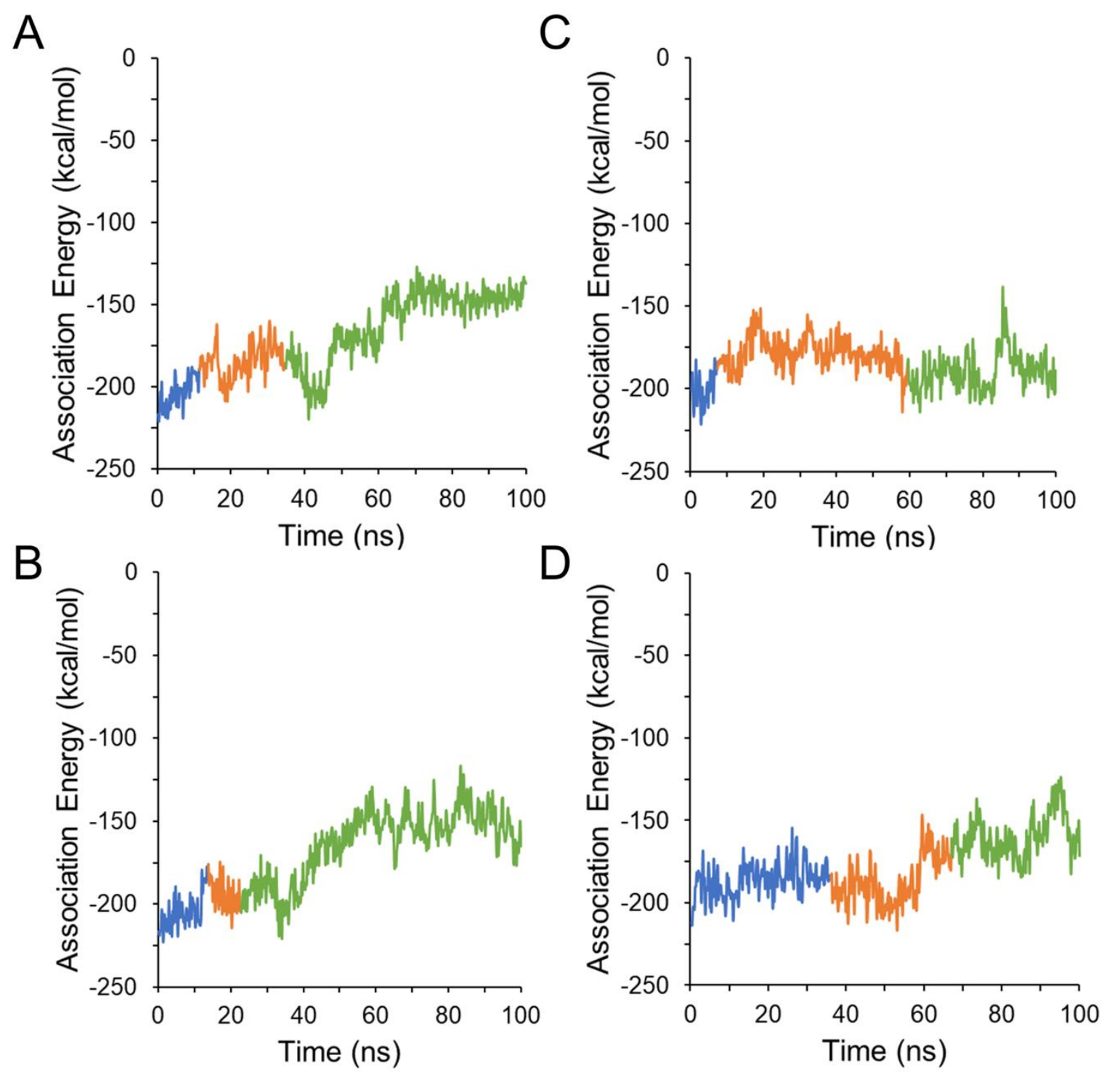

Figure S16: The association energy (calculated through MM-GBSA approximation) between the first outermost peptide and the rest of the $A \beta_{1-42}$ fibril as a function of simulation time for (A) SY12, (B) SY31, (C) SY5, and (D) curcumin. The plotted association energy was calculated from simulations in which the molecules acquire the lowest average binding energy, per molecule, and adopt binding mode $p d 1$ and correspond to the same simulations analyzed in Figure S15. Blue represents the association energy prior to the simulation stage leading to partial dissociation corresponding to the respective simulation. Orange represents the association energy during the simulation stage leading to partial dissociation. Green represents the association energy posterior to the simulation stage leading to partial dissociation. 


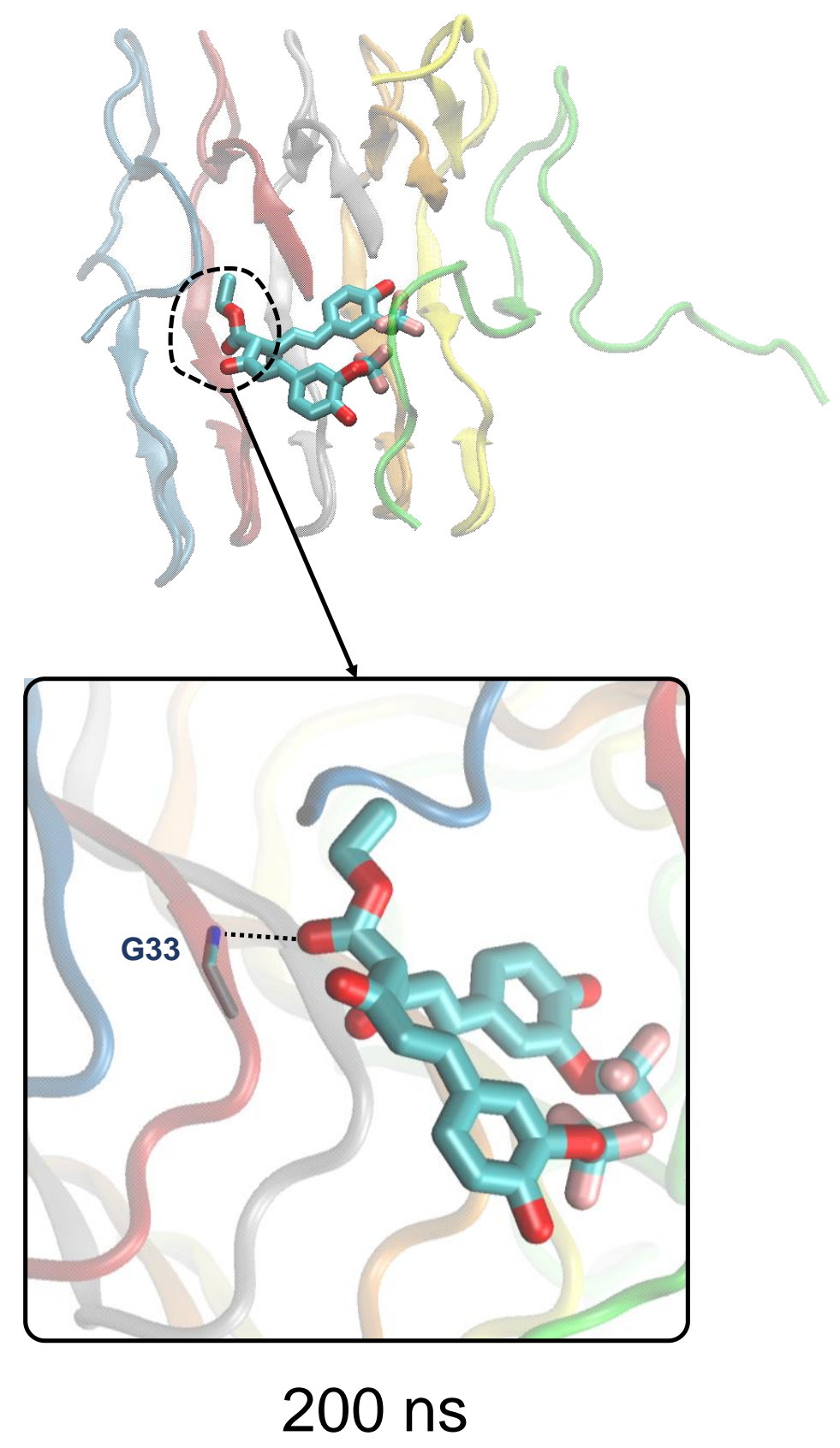

Figure S17: Molecular graphics image of SY12 partially dissociating the $A \beta_{1-42}$ fibril after adopting the lowest energy binding mode $p d 3$ at 200 ns. SY12 is shown in thick licorice representation, the $A \beta_{1-42}$ fibril is shown in cartoon representation. Bird's eye views of SY12 in complex with the disrupted $\mathrm{A} \beta_{1-42}$ fibril at $200 \mathrm{~ns}$ (top). SY12 remains in the binding mode 3 conformation potentially due to its ability to form hydrogen bonds to the backbone $\mathrm{N}$ of Gly ${ }^{33}$ within the $A \beta_{1-42}$ fibril through the anchoring $R^{1}$ group, indicated by a black dotted line (bottom). 
<smiles>C=CCC(=O)C=Cc1ccc(O)c(OC)c1</smiles><smiles>COc1cc(/C=C/C(=O)/C=C(O)/C=C/c2ccc(O)c(OC)c2)ccc1O</smiles>

Figure S18: Structure of curcumin's keto-form (top) and curcumin's enol-form (bottom). 

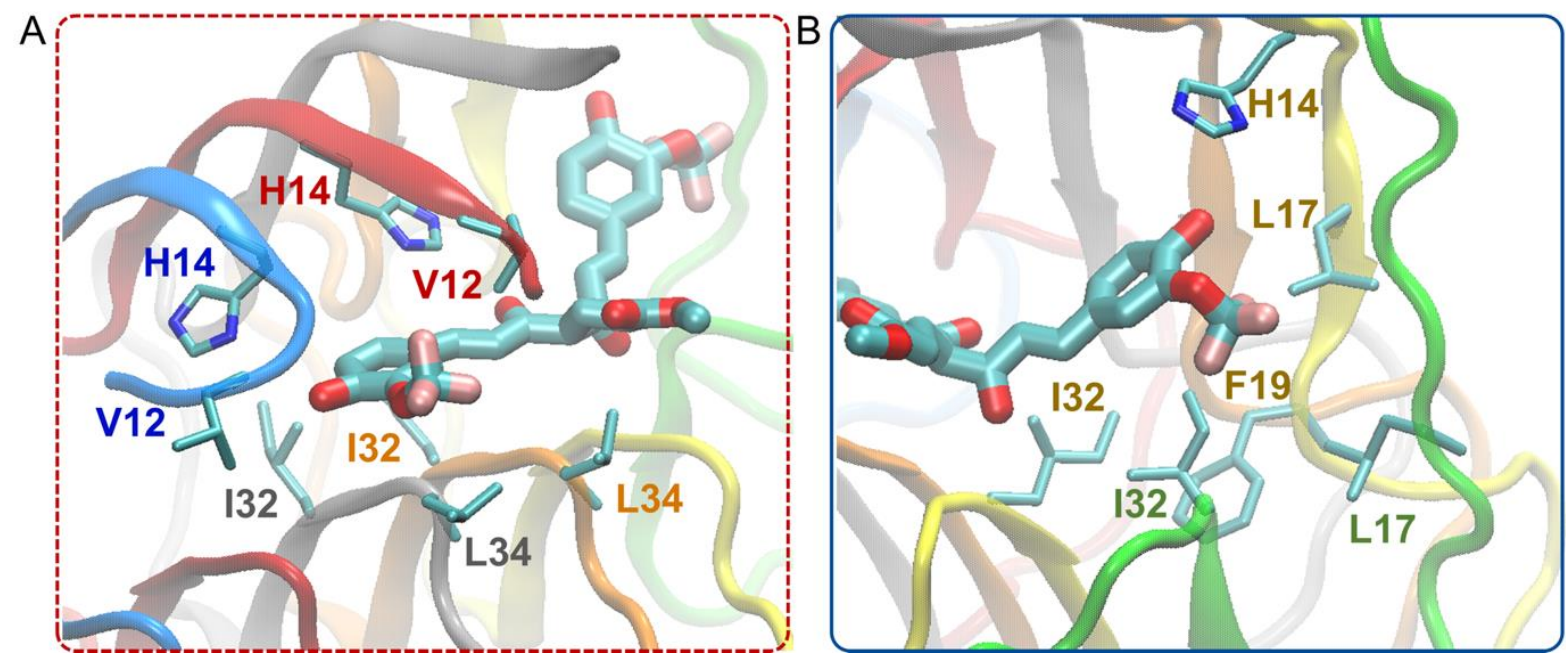

Figure S19: Molecular graphics images of SY5 in complex with $A \beta_{1-42}$ fibril that shifted and adopted binding mode $p d 1$ after $100 \mathrm{~ns}$ of simulation time. SY5 is shown in thick licorice representation, the $A \beta_{1-42}$ fibril is shown in cartoon representation, and the $A \beta_{1-42}$ residues are shown in licorice representation. (A) Key interactions between the anchor group and A $\beta_{1-42}$ residues stabilizing the SY5 molecule. (B) Key interactions between the breaker group and $A \beta_{1-42}$ residues destabilizing the $A \beta_{1-42}$ fibril. In the simulation in which SY5 adopted the lowest energy binding mode $n d 1$, SY5 shifted to adopt binding mode $p d 1$ after 100 ns. SY5 then interacted with the residues highlighted in (A) and (B), leading to partial dissociation. This validates the characterization of $A \beta_{1-42}$ residues acting as switches for partial dissociation. 

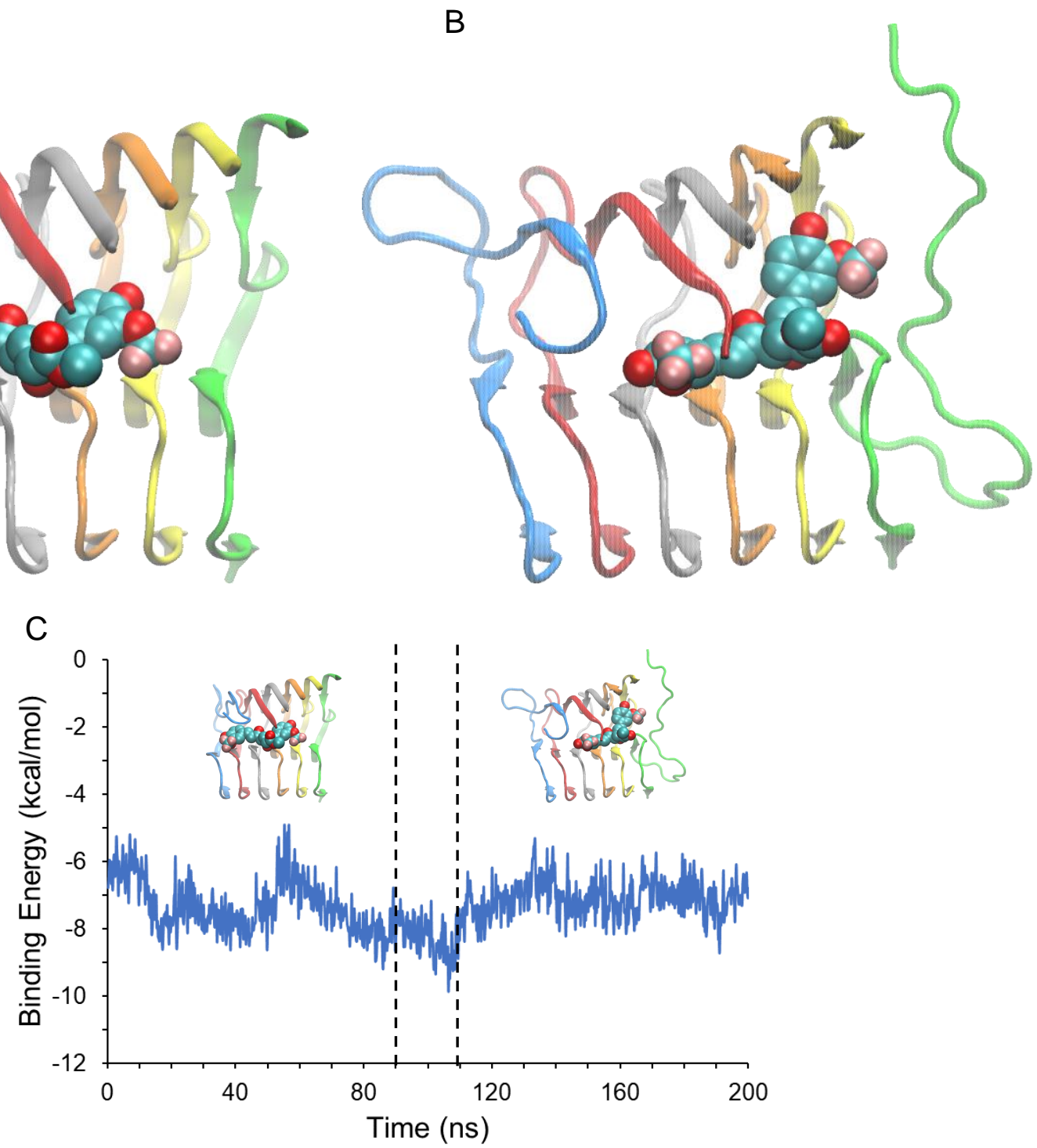

Figure S20: Molecular graphics images of SY5 in complex with $A \beta_{1-42}$ fibril that adopted the lowest energy binding mode $p d 1$ (amongst the simulations of SY 5 in complex with $\mathrm{A} \beta_{1-42}$ fibril) after $100 \mathrm{~ns}$ of simulation time when the SY5 initially adopted binding mode $n d 1$. SY5 is shown in van der Waals representation and the $A \beta_{1-42}$ fibril are shown in cartoon representation. (A) Bird's eye view of SY5 adopting binding mode $n d 1$ prior to $100 \mathrm{~ns}$ of simulation time. (B) Bird's eye view of SY5 adopting binding mode pd1 after 100 ns of simulation time. (C) The binding energy (calculated through AutoDock Vina) between SY5 and the $A \beta_{1-42}$ fibril as a function of simulation time. The molecule shifts from binding mode $n d 1$ to binding mode $p d 1$ between $90 \mathrm{~ns}$ and $110 \mathrm{~ns}$ of simulation time as indicated by the black, dotted lines in (C). This shift is characterized by a decrease in binding energy. 


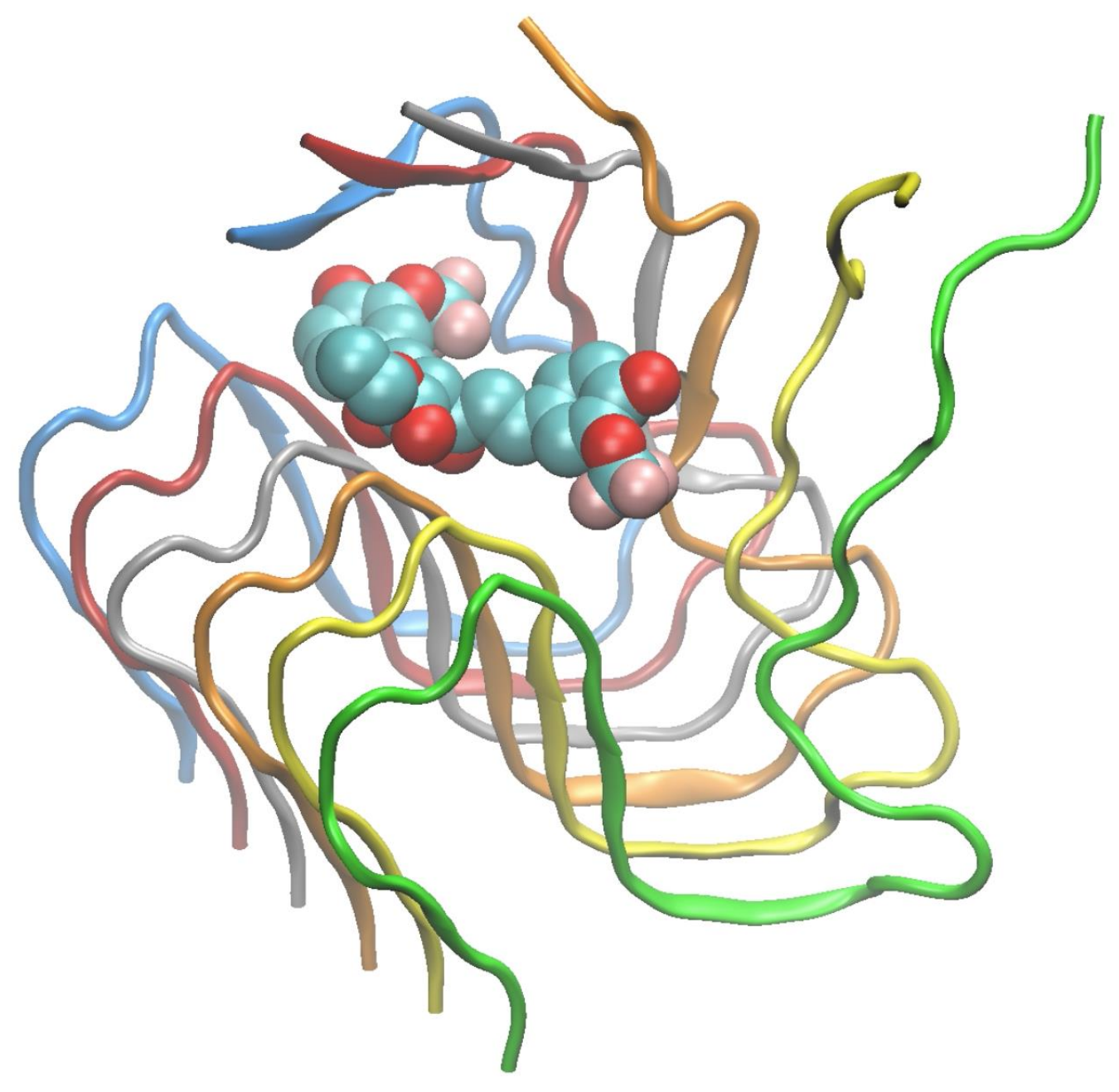

Movie S1. Molecular graphics movie of SY12 dissociating the $A \beta_{1-42}$ fibril after adopting binding mode $p d 1 . \mathrm{SY} 12$ is shown in $\mathrm{VdW}$ representation, the $\mathrm{A} \beta_{1-42}$ fibril is shown in cartoon representation. The partly dissociated outermost peptide is shown in green cartoon representation. The wrapping of the partly dissociated outermost peptide's ${ }_{16} \mathrm{KLVFF}_{20}$ domain to form intramolecular interactions with the same peptide's ${ }_{29} \mathrm{GAIIG}_{33}$ domain can be seen at 0:21 in the video (corresponding to $\sim 90 \mathrm{~ns}$ ). The patching of the KLVFF domain can be seen at 0:24 in the video (corresponding to $\sim 100 \mathrm{~ns}$ ). 
Table S1: Average binding energy between the molecules and the modeled $A \beta_{1-42}$ fibril corresponding to the lowest energy binding modes 1, 2 and 3, not leading to partial dissociation $(n d)$, compared to those leading to partial dissociation ( $p d$ ), calculated using AutoDock Vina scoring function. The binding energies were averaged over the snapshots of the entire simulation trajectory for each simulated binding complex. Average binding energies for simulated binding modes that do not occur for a particular molecule are not applicable and are denoted as n. a.

\begin{tabular}{|c|c|c|c|c|c|c|}
\hline \multirow{2}{*}{$\begin{array}{c}\text { AutoDock Vina } \\
\text { Binding Affinity } \\
\left(\mathbf{k c a l ~ m o l}^{-1}\right)\end{array}$} & \begin{tabular}{c} 
Binding mode \\
\cline { 2 - 7 }
\end{tabular} & $\begin{array}{c}\text { Binding mode } \\
\boldsymbol{n d 1}\end{array}$ & $\begin{array}{c}\text { Binding mode } \\
\boldsymbol{p d 2}\end{array}$ & $\begin{array}{c}\text { Binding mode } \\
\boldsymbol{n d 2}\end{array}$ & $\begin{array}{c}\text { Binding mode } \\
\boldsymbol{p d 3}\end{array}$ & $\begin{array}{c}\text { Binding mode } \\
\boldsymbol{n d 3}\end{array}$ \\
\hline SY12 & $-8.0 \pm 0.7$ & $-7.1 \pm 0.5$ & $-5.6 \pm 0.9$ & $-7.1 \pm 0.8$ & $-5.5 \pm 0.8$ & $-5.9 \pm 1.0$ \\
\hline SY31 & $-7.1 \pm 1.2$ & $-7.5 \pm 0.6$ & $-7.1 \pm 0.7$ & $-7.5 \pm 0.6$ & $-5.2 \pm 0.7$ & $-5.4 \pm 0.8$ \\
\hline SY5 & $-8.2 \pm 0.8$ & $-7.4 \pm 0.9$ & $-6.8 \pm 0.8$ & $-6.1 \pm 0.6$ & n. a. & $-6.4 \pm 0.9$ \\
\hline Curcumin & $-6.2 \pm 0.7$ & $-6.7 \pm 0.5$ & n. a. & n. a. & n. a. & n. a. \\
\hline
\end{tabular}

Table S2: Average binding energy between the molecules and the modeled $A \beta_{1-42}$ fibril corresponding to the simulations in which the molecules adopted the lowest energy binding mode $p d 1$, calculated using AutoDock Vina scoring function. The binding energies were calculated per snapshot and averaged over the entire simulation trajectory for each simulated binding complex prior to the respective simulation stage, during the simulation stage, and posterior to the simulation stage.

\begin{tabular}{|c|c|c|c|}
\hline $\begin{array}{c}\text { AutoDock Vina Binding } \\
\text { Affinity }\left(\mathbf{k c a l ~ m o l}^{-\mathbf{1}}\right)\end{array}$ & $\begin{array}{c}\text { Prior to } \\
\text { simulation stage }\end{array}$ & $\begin{array}{c}\text { During simulation } \\
\text { stage }\end{array}$ & $\begin{array}{c}\text { Posterior to } \\
\text { simulation stage }\end{array}$ \\
\hline SY12 & $-8.0 \pm 0.6$ & $-8.1 \pm 0.5$ & $-7.9 \pm 0.6$ \\
\hline SY31 & $-6.3 \pm 0.5$ & $-5.7 \pm 0.5$ & $-7.4 \pm 1.2$ \\
\hline SY5 & $-6.8 \pm 0.6$ & $-8.5 \pm 0.5$ & $-8.2 \pm 0.4$ \\
\hline Curcumin & $-5.9 \pm 0.7$ & $-6.6 \pm 0.6$ & $-6.3 \pm 0.5$ \\
\hline
\end{tabular}


Supporting initial structures are provided in PDB format. The initial structures used for each simulation for each compound are provided as Supporting Material in PDB format. The structures are superimposed with respect to the backbone of the entire modeled $A \beta_{1-42}$ fibril. The correspondence of PDB files, the molecules in complex with the $A \beta_{1-42}$ fibril, and the number of the MD simulations from which the initial structures originate are shown in the table.

\begin{tabular}{|c|c|c|}
\hline PDB file & $\begin{array}{c}\text { Molecule in complex } \\
\text { with } \mathbf{A}_{\mathbf{1}-\mathbf{4 2}} \text { fibril }\end{array}$ & $\begin{array}{c}\text { Initial Structures } \\
\text { for Simulations }\end{array}$ \\
\hline initial.sy12.pdb & SY12 & $1-25$ \\
\hline initial.sy31.pdb & SY31 & $1-25$ \\
\hline initial.sy5.pdb & SY5 & $1-23$ \\
\hline initial.curcumin.pdb & Curcumin & $1-21$ \\
\hline
\end{tabular}




\section{Supporting References}

${ }^{1}$ Jorgensen, W. L.; Chandrasekhar, J.; Madura, J. D.; Impey, R. W.; Klein, M. L., Comparison of simple potential functions for simulating liquid water. The Journal of Chemical Physics 1983, 79 (2), 926-935.

${ }^{2} \mathrm{Li}$, S.; Hong, M., Protonation, tautomerization, and rotameric structure of histidine: a comprehensive study by magic-angle-spinning solid-state NMR. J Am Chem Soc 2011, 133 (5), 1534-1544.

${ }^{3}$ Jo, S.; Kim, T.; Iyer, V. G.; Im, W. CHARMM-GUI: A WebBased Graphical User Interface for CHARMM. J. Comput. Chem. 2008, 29, 1859-1865.

${ }^{4}$ Lodish, H.; Berk, A.; Zipursky, S. L.; Matsudaira, P.; Baltimore, D.; Darnell, J. E. Molecular Cell Biology; W. H. Freeman: New York, 2000.

${ }^{5}$ LaFerla F. M.; Green, K. N.; Oddo, S., Intracellular amyloid-beta in Alzheimer's disease. Nat Rev Neurosci. 2007, $8(7), 499-509$.

${ }^{6}$ Brooks, B. R.; Brooks, C. L., 3rd; MacKerell, A. D., Jr.; Nilsson, L.; Petrella, R. J.; Roux, B.; Won, Y.; Archontis, G.; Bartels, C.; Boresch, S.; et al. CHARMM: The Biomolecular Simulation Program. J. Comput. Chem. 2009, 30, $1545-1614$.

${ }^{7}$ Im, W.; Lee, M. S.; Brooks, C. L. 3rd. Generalized born model with a simple smoothing function. J. Comput. Chem. 2003, 24, 1691-1702.

${ }^{8}$ Hayes, J. M.; Archontis, G. MM-GB (PB) SA Calculations of Protein-Ligand Binding Free Energies. In Molecular Dynamics - Studies of Synthetic and Biological Macromolecules; Wang, L., Ed.; InTech: Rijeka, Croatia, 2012.

${ }^{9}$ Xiao, Y.; Ma, B.; McElheny, D.; Parthasarathy, S.; Long, F.; Hoshi, M.; Nussinov, R.; Ishii, Y., A $\beta(1-42)$ fibril structure illuminates self-recognition and replication of amyloid in Alzheimer's disease. Nature structural \& molecular biology 2015, 22 (6), 499-505.

${ }^{10}$ Kollman, P. A.; Massova, I.; Reyes, C.; Kuhn, B.; Huo, S.; Chong, L.; Lee, M.; Lee, T.; Duan, Y.; Wang, W.; Donini, O.; Cieplak, P.; Srinivasan, J.; Case, D. A.; Cheatham, T. E., Calculating Structures and Free Energies of Complex Molecules: Combining Molecular Mechanics and Continuum Models. Accounts of Chemical Research 2000, 33 (12), 889-897.

11 Trott, O.; Olson, A. J., AutoDock Vina: improving the speed and accuracy of docking with a new scoring function, efficient optimization, and multithreading. Journal of Computational Chemistry 2010, 31 (2), $455-461$. 